\title{
A New Lifetime Distribution and Its Power Transformation
}

\author{
Ammar M. Sarhan, ${ }^{1}$ Lotfi Tadj, ${ }^{2}$ and David C. Hamilton ${ }^{1}$ \\ ${ }^{1}$ Department of Mathematics, Dalhousie University, Halifax, NS, Canada B3H 4R2 \\ ${ }^{2}$ Department of Information Systems and Decision Sciences, Fairleigh Dickinson University, Vancouver, BC, Canada V6B 2 P6
}

Correspondence should be addressed to Ammar M. Sarhan; asarhan0@yahoo.com

Received 11 January 2014; Revised 8 April 2014; Accepted 12 April 2014; Published 18 May 2014

Academic Editor: Chin-Shang Li

Copyright (C) 2014 Ammar M. Sarhan et al. This is an open access article distributed under the Creative Commons Attribution License, which permits unrestricted use, distribution, and reproduction in any medium, provided the original work is properly cited.

\begin{abstract}
New one-parameter and two-parameter distributions are introduced in this paper. The failure rate of the one-parameter distribution is unimodal (upside-down bathtub), while the failure rate of the two-parameter distribution can be decreasing, increasing, unimodal, increasing-decreasing-increasing, or decreasing-increasing-decreasing, depending on the values of its two parameters. The two-parameter distribution is derived from the one-parameter distribution by using a power transformation. We discuss some properties of these two distributions, such as the behavior of the failure rate function, the probability density function, the moments, skewness, and kurtosis, and limiting distributions of order statistics. Maximum likelihood estimation for the two-parameter model using complete samples is investigated. Different algorithms for generating random samples from the two new models are given. Applications to real data are discussed and compared with the fit attained by some one- and two-parameter distributions. Finally, a simulation study is carried out to investigate the mean square error of the maximum likelihood estimators, the coverage probability, and the width of the confidence intervals of the unknown parameters.
\end{abstract}

\section{Introduction}

Lindley [1] proposed a one-parameter distribution, now known as the Lindley distribution, with the following probability density function (pdf):

$$
f(t)=\frac{\beta^{2}}{\beta+1}(1+t) e^{-\beta t}, \quad t>0, \beta>0 .
$$

The failure rate function of the Lindley distribution is always increasing. The properties of the Lindley distribution are studied in detail by Ghitany et al. [2]. There are situations in which the Lindley distribution may not be suitable from a theoretical or applied point of view, Ghitany et al. [3]. For this reason, Ghitany et al. [3] used a power transformation, $X=T^{1 / \alpha}$, to introduce the power Lindley distribution which is a more flexible distribution. The $\operatorname{pdf}$ of $\operatorname{PL}(\alpha, \beta)$ is

$$
f(x)=\frac{\alpha \beta^{2}}{\beta+1}\left(1+x^{\alpha}\right) e^{-\beta x^{\alpha}}, \quad x>0, \alpha, \beta>0 .
$$

Ghitany et al. [3] showed that the hazard function of $\operatorname{PL}(\alpha, \beta)$ can be increasing, decreasing, and decreasing-increasingdecreasing depending on the values of the parameters. They also discussed some of the statistical properties of the distribution and used the maximum likelihood method to estimate its two unknown parameters and applied it to a real data set. In spite of the flexibility of the $\operatorname{PL}(\alpha, \beta)$ to fit some real data sets, it fails to fit some other data sets.

The main aim of this paper is to introduce two new distributions. The first is a one-parameter distribution which is similar to the Lindley distribution and the second is the power transformation of the one-parameter distribution. We refer to these two distributions as $N(\beta)$ and $\operatorname{TN}(\alpha, \beta)$ respectively. The hazard function of $N(\beta)$ is only unimodal, while the hazard function of $\operatorname{TN}(\alpha, \beta)$ can be decreasing, increasing, unimodal, decreasing-increasingdecreasing, or increasing-decreasing-increasing depending on the values of its two parameters. The variety of shapes of the hazard function of the $\operatorname{TN}(\alpha, \beta)$ enables it to be a good model to fit different data sets. 
The rest of the paper is organized as follows. Section 2 introduces the new one-parameter distribution and some of its characteristics are discussed in Section 3. Section 4 presents the transformation of the new distribution, $\mathrm{TN}(\alpha, \beta)$. Different characteristics of $\mathrm{TN}(\alpha, \beta)$, such as the hazard function, quantiles, random sample generation, moments, and order statistics distributions, are discussed in Section 5. Section 6 discusses the maximum likelihood estimate of the two parameters of $\operatorname{TN}(\alpha, \beta)$. Applications of the two models are presented in Section 6. Monte Carlo Simulation study is carried out in Section 7 to examine the accuracy of the maximum likelihood estimators of the $\mathrm{TN}(\alpha, \beta)$ parameters as well as the coverage probability and average width of the confidence intervals for the parameters. Finally, Section 8 concludes this paper.

\section{The New Distribution}

Consider the random variable $T$ whose pdf is given by

$$
f(t)=\frac{\beta}{1+\beta}\left[\beta+(1+2 \beta t) e^{-\beta t}\right] e^{-\beta t}, \quad t \geq 0, \beta>0 .
$$

The survival function (sf) of $T$ is given by

$$
S(t)=\frac{1}{1+\beta}\left[\beta+(1+\beta t) e^{-\beta t}\right] e^{-\beta t}, \quad t \geq 0, \beta>0,
$$

while its hazard rate function is given by

$$
h(t)=\frac{\beta\left[\beta+(1+2 \beta x) e^{-\beta t}\right]}{\beta+(1+\beta t) e^{-\beta t}}, \quad t \geq 0, \beta>0 .
$$

For simplicity, from now on, we refer to this distribution as $N(\beta)$.

Interpretation. There are two different interpretations of $N(\beta)$ as follows.

(1) The pdf $f(t)$ is a mixture density of two mixture components. One follows $\operatorname{Exp}(\beta)$ and the other is the lifetime of a two independent component series system with $\operatorname{Exp}(\beta)$ and $G(2, \beta)$ and mixture weights $a_{1}=\beta /(1+\beta)$ and $a_{2}=1 /(1+\beta)$, respectively. This means that $N(\beta)$ can be expressed in terms of $\operatorname{Exp}(\beta)$ and $G(2, \beta)$ as $N(\beta)=a_{1} \operatorname{Exp}(\beta)+$ $a_{2} \min \{\operatorname{Exp}(\beta), G(2, \beta)\}$.

(2) The random variable $T$ can be described as a mixture of three components: $\operatorname{Exp}(\beta), \operatorname{Exp}(2 \beta)$, and a $G(2,2 \beta)$ with mixture weights $b_{1}=\beta /(1+$ $\beta), b_{2}=1 / 2(1+\beta)$ and $b_{3}=1 / 2(1+\beta)$, respectively. This means that $N(\beta)$ can be expressed in terms of $\operatorname{Exp}(\beta), \operatorname{Exp}(2 \beta)$ and $G(2,2 \beta)$ as $N(\beta)=$ $b_{1} \operatorname{Exp}(\beta)+b_{2} \operatorname{Exp}(2 \beta)+b_{3} G(2,2 \beta)$.

Some characteristics of $N(\beta)$ are derived in the next section.

\section{Characteristics of $N(\beta)$}

In this section, algorithms are described to obtain quantiles of $N(\beta)$ and to generate samples from $N(\beta)$. Also, the moment generating function and the moments of this distribution are derived.

3.1. Quantiles. The $100 q$ th quantile, $q \in(0,1)$, can be derived as follows.

(1) Let $q \in(0,1)$;

(2) Solve the following equation numerically in $u \in$ $(0,1)$ :

$$
[\beta+(1-\ln u) u] u-(1+\beta)(1-q)=0
$$

(3) The $100 q$ th quantile is $t_{q}=-\ln u / \beta$.

3.2. Random Sample Generation. We provide below three equivalent algorithms to generate a random variate from $N(\beta)$.

Algorithm 1. (1) Generate $u \sim U(0,1)$.

(2) Solve numerically the following equation in $v \in$ $(0,1):[\beta+(1-\ln v) v] v-(1+\beta) u=0$.

(3) Set $T=-\ln v / \beta$.

Algorithm 2. (1) Generate I from the set $\{1,2\}$ such that $P(I=j)=a_{j}, j=1,2$.

(a) If $I=1$, set $y_{1}=-\ln u / \beta$, where $u \sim U(0,1)$.

(b) If $I=2$, set $y_{2}=\min \left\{-\ln u_{1} / \beta,-\ln u_{2} u_{3} / \beta\right\}$, where $u_{1}, u_{2}, u_{3} \sim U(0,1)$.

(2) Set $T=y_{I}$.

Algorithm 3. (1) Generate I from the set $\{1,2,3\}$ such that $P(I=j)=b_{j}, j=1,2,3$.

(a) If $I=1$, set $y_{1}=-\ln u / \beta$, where $u \sim U(0,1)$.

(b) If $I=2$, set $y_{2}=-\ln u / 2 \beta$, where $u \sim U(0,1)$.

(c) If $I=3$, set $y_{3}=-\ln u_{1} u_{2} / 2 \beta$, where $u_{1}, u_{2} \sim$ $U(0,1)$.

(2) Set $T=y_{I}$.

3.3. The Moments and the Moment Generating Function. The moment generating function (mgf) of $N(\beta)$ may be written as

$$
M(t)=\frac{\beta}{1+\beta}\left[\frac{\beta}{\beta-t}+\frac{4 \beta-t}{(2 \beta-t)^{2}}\right], \quad t<\beta .
$$

Differentiating the above expression $k$ times with respect to $t$ and setting $t$ to zero, we get $k$ th moments, $\mu_{k}$, as

$$
\mu_{k}=E\left(T^{k}\right)=\frac{\beta}{1+\beta}\left[1+\frac{\beta}{2 \beta}\left(1+\frac{k+1}{2 \beta}\right) \frac{1}{2^{k}}\right] \frac{k !}{\beta^{k}}, \quad k \geq 1 .
$$



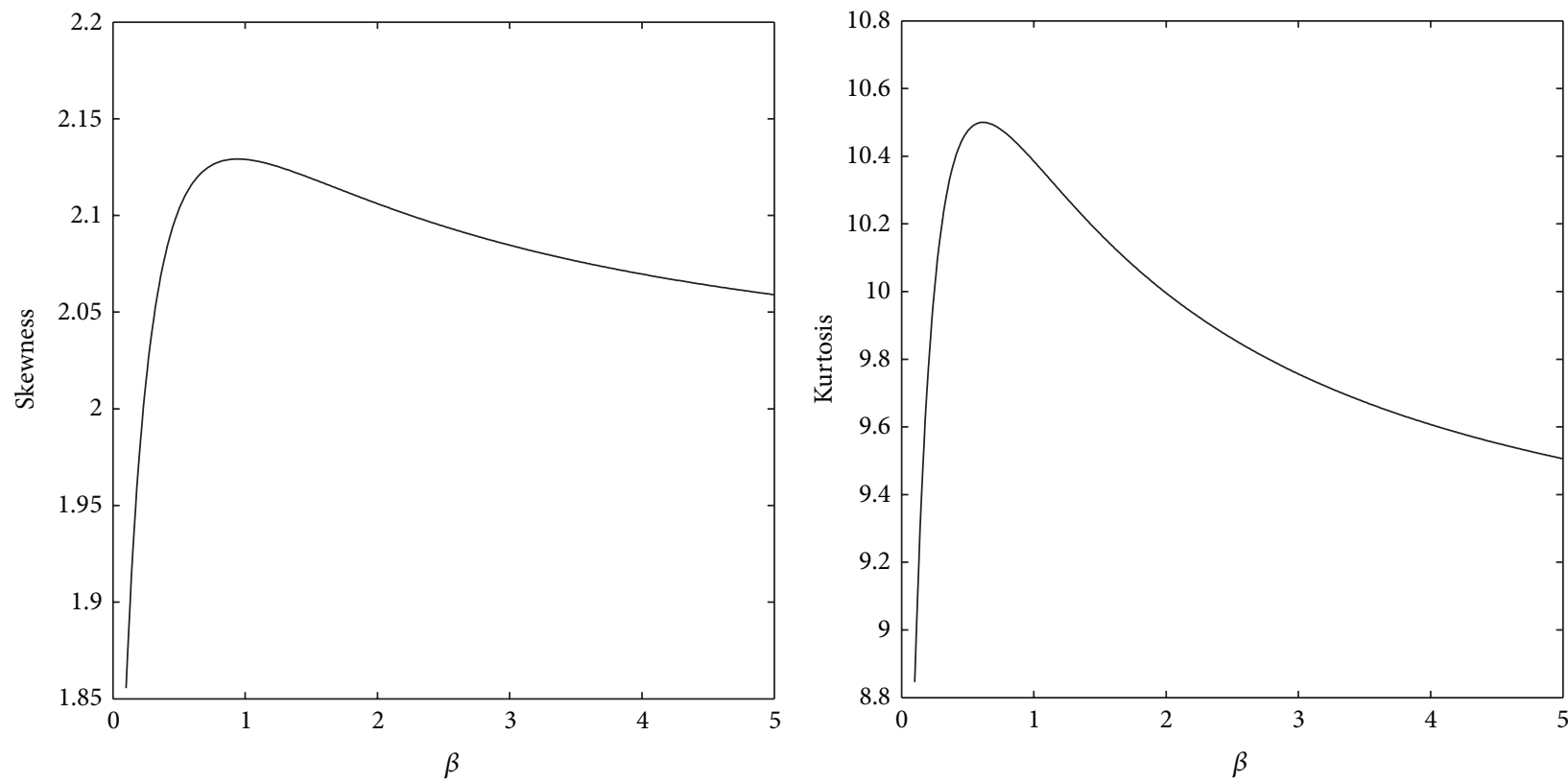

Figure 1: Skewness and kurtosis of $N(\beta)$ as functions $\beta$.

Based on the first four ordinary moments, the measures of skewness (sk) and kurtosis $(\kappa)$ of $N(\beta)$ can be obtained using

$$
\begin{gathered}
\text { sk }=\frac{\mu_{3}-3 \mu_{1} \mu_{2}+2 \mu_{1}^{3}}{\left[\mu_{2}-\mu_{1}^{2}\right]^{3 / 2}}, \\
\kappa=\frac{\mu_{4}-4 \mu_{1} \mu_{3}+6 \mu_{1}^{2} \mu_{2}-3 \mu_{1}^{4}}{\left[\mu_{2}-\mu_{1}^{2}\right]^{2}} .
\end{gathered}
$$

Plots of the skewness and kurtosis of the distribution as a function of $\beta$ are plotted in Figure 1. From the plots, sk and $\kappa$ are unimodal functions of $\beta$. The skewness is always positive and the kurtosis is larger than 3; therefore, $N(\beta)$ is positively skewed and leptokurtic.

\section{Power Transformation of the New Distribution}

To get a more flexible distribution, we consider an extension of the new distribution $N(\beta)$ with the pdf (3) by using the power transformation $X=T^{1 / \alpha}, \alpha>0$. The pdf of $X$ is given by

$$
\begin{array}{r}
f(x)=\frac{\alpha \beta x^{\alpha-1}}{1+\beta}\left[\beta+\left(1+2 \beta x^{\alpha}\right) e^{-\beta x^{\alpha}}\right] e^{-\beta x^{\alpha}} \\
x \geq 0, \quad \alpha, \beta>0 .
\end{array}
$$

The density of $X$ is plotted in Figure 2 for three choices of $\alpha$ when $\beta=1.0$, which shows that the density is symmetric when $\alpha=3.535$, left skewed when $\alpha<3.535$, and right skewed when $\alpha>3.535$. This implies that the power parameter $\alpha$ characterizes the shape of the density function. More investigation of the density will be discussed, in the next section, based on the skewness and kurtosis measures. From now on, we will use $\operatorname{TN}(\alpha, \beta)$ to refer to the power transformation of the new distribution $N(\beta)$.

Interpretation. There are two different interpretations of $Y$ as follows.

(1) The pdf $f(x)$ is a mixture density of two mixture components. One component follows $W(\alpha, \beta)$ and the other is the lifetime of a two independent component series system with $W(\alpha, 2 \beta)$ and $\operatorname{PG}(\alpha, 2, \beta)$ and mixture weights $a_{1}=\beta /(1+\beta)$ and $a_{2}=1 /(1+\beta)$, respectively.

(2) The random variable $X$ can be described as a mixture of three components: $W(\alpha, \beta), W(\alpha, 2 \beta)$, and a $\operatorname{PG}(\alpha, 2,2 \beta)$ with mixture weights $b_{1}=\beta /(1+\beta), b_{2}=$ $1 / 2(1+\beta)$, and $b_{3}=1 / 2(1+\beta)$, respectively.

Straightforward calculations yield the the survival function of $\operatorname{TN}(\alpha, \beta)$ as

$$
\begin{array}{r}
S(x)=\frac{1}{1+\beta}\left[\beta+\left(1+\beta x^{\alpha}\right) e^{-\beta x^{\alpha}}\right] e^{-\beta x^{\alpha}}, \\
x \geq 0, \quad \alpha, \beta>0 .
\end{array}
$$

We derive some characteristics of $\operatorname{TN}(\alpha, \beta)$ in the next section. 


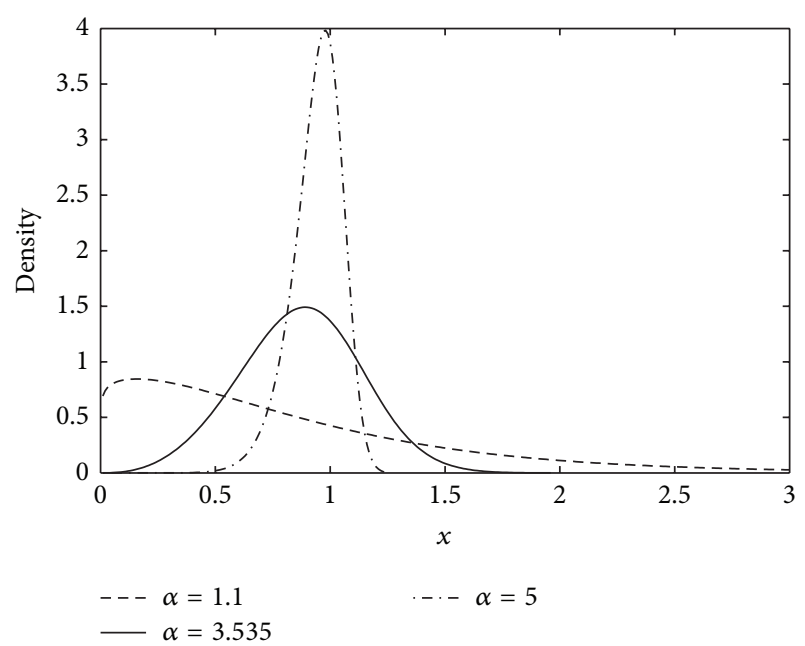

FIgURE 2: The $\operatorname{TN}(\alpha, \beta)$ density function for some values of $\alpha$ when $\beta=1$.

\section{Characteristics of $\operatorname{TN}(\alpha, \beta)$}

5.1. The Hazard Function. The hazard rate function of $\mathrm{TN}(\alpha, \beta)$ is

$$
h(x)=\frac{\alpha \beta x^{\alpha-1}\left[\beta+\left(1+2 \beta x^{\alpha}\right) e^{-\beta x^{\alpha}}\right]}{\beta+\left(1+\beta x^{\alpha}\right) e^{-\beta x^{\alpha}}}, \quad x \geq 0, \alpha, \beta>0 .
$$

For $\alpha=1$, the hazard function is unimodal. Its limiting values at zero and infinity are $\beta$, and it reaches a maximum value of

$$
\frac{\left(1+2 W_{-1}\left(e^{-1} / \beta\right)\right) \beta}{1+W_{-1}\left(e^{-1} / \beta\right)}
$$

at

$$
\frac{1}{\beta}\left(1+W_{-1}\left(\frac{e^{-1}}{\beta}\right)\right)
$$

where $W_{-1}(\cdot)$ denotes the Lambert $W$ function which is the inverse of the function $x e^{x}$.

For $\alpha \neq 1$, the shape of the hazard function is difficult to ascertain analytically. The shape was determined numerically by examining the derivative of the hazard out to the 99.99th percentile of the distribution, and the results are shown in Figure 3. For $\alpha<1$, the hazard is decreasing except for a small region with $\alpha$ close to 1 and $\beta<0.5$ where the hazard is initially decreasing, then increasing, and finally decreasing (DID). For $\alpha>1$, the hazard is strictly increasing for large $\beta$ ( $\beta>2.6$ in the figure). For smaller $\beta$ with $\alpha$ close to 1 , the hazard can be unimodal (for very small $\beta$ ) or initially increasing, then decreasing, and finally increasing (IDI) (for slightly larger $\beta$ ). Figure 4 shows the hazard for five choices of $\alpha$ and $\beta$ which demonstrate the five possible shapes.

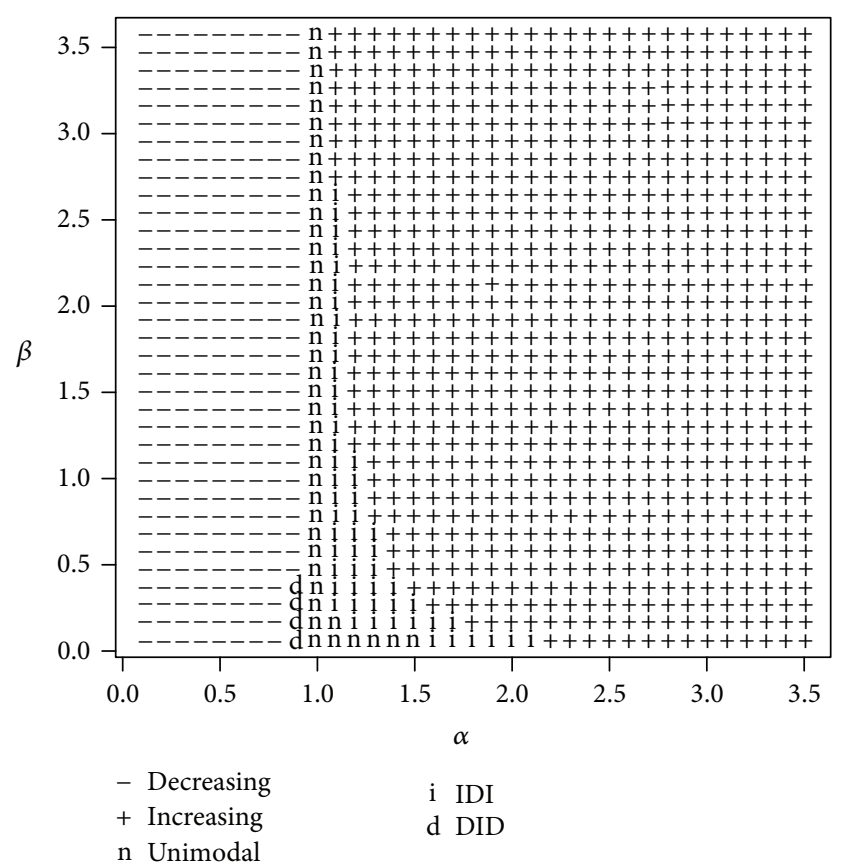

FIgURE 3: Shapes of the hazard function of $\operatorname{TN}(\alpha, \beta)$.

5.2. Quantiles and Random Sample Generations. The $100 q$ th quantile of $\operatorname{TN}(\alpha, \beta), x_{q}$, can be derived from that of $N(\beta), t_{q}$, as follows:

$$
x_{q}=t_{q}^{1 / \alpha}
$$

Figure 5 depicts the three quartiles $Q_{1}, Q_{2}$, and $Q_{3}$, which can be obtained from the $q$ th quantile by setting $q=0.25,0.50$, and 0.75 , respectively. From Figure 5, the Interquartile range $\left(\mathrm{IQR}=Q_{3}-Q_{1}\right)$ decreases dramatically when $\alpha$ increases.

The following algorithm generates a random variate from $\operatorname{TN}(\alpha, \beta)$.

Algorithm 4. (1) Generate $T$ from $N(\beta)$, using one of the Algorithms 1-3;

(2) Set $X=T^{1 / \alpha}$.

5.3. The Moments and Shape Measures. Let $X$ follow $\mathrm{TN}(\alpha, \beta)$. After some algebra, the $k$ th ordinary moment of $X$ is derived as

$$
\begin{aligned}
\mu_{k} & =E\left(X^{k}\right) \\
& =b_{1} \frac{\Gamma(k / \alpha+1)}{\beta^{k / \alpha}}+b_{1} \frac{\Gamma(k / \alpha+1)}{(2 \beta)^{k / \alpha}}+b_{3} \frac{\Gamma(k / \alpha+2)}{(2 \beta)^{k / \alpha}} \\
& =\frac{k \Gamma(k / \alpha)\left[2 \alpha\left(1+2^{k / \alpha} \beta\right)+k\right]}{2 \alpha^{2}(1+\beta)(2 \beta)^{k / \alpha}} .
\end{aligned}
$$


Therefore, the mean and variance of $X$ are

$$
\begin{gathered}
\mu=\frac{\Gamma(1 / \alpha)\left[2 \alpha\left(1+2^{1 / \alpha} \beta\right)+1\right]}{2 \alpha^{2}(1+\beta)(2 \beta)^{1 / \alpha}}, \\
\sigma^{2}=\left(8 \alpha^{2}(1+\beta) \Gamma\left(\frac{2}{\alpha}\right)\left[\alpha\left(1+2^{2 / \alpha} \beta\right)+1\right]-\Gamma^{2}\left(\frac{1}{\alpha}\right)\right. \\
\left.\times\left[2 \alpha\left(1+2^{1 / \alpha} \beta\right)+1\right]^{2}\right) \\
\times\left(4 \alpha^{4}(1+\beta)^{2}(2 \beta)^{2 / \alpha}\right)^{-1} .
\end{gathered}
$$

Figure 6 depicts the mean and variance of $\operatorname{TN}(\alpha, \beta)$ as functions of $\alpha$ when $\beta=1$ which shows that the mean decreases dramatically in $\alpha$ and takes its minimum of 0.8298 at $\alpha=1.61$ then it increases steadily to take its maximum of 0.9687 , while the variance is decreasing.

Based on the first four ordinary moments, the measures of skewness (sk) and kurtosis $(\kappa)$ of $\mathrm{TN}(\alpha, \beta)$ can be obtained by substituting (17) into (9) and (10), respectively. Plots of the skewness and kurtosis of $\operatorname{TN}(\alpha, \beta)$ distribution as functions of $\alpha$, when $\beta=1.0$, are given in Figure 7. From these plots, (1) the skewness is positive when $\alpha<3.535$ and negative when $\alpha>3.535$ and the kurtosis is (i) equal to 3 when either $\alpha=2.4977$ or $\alpha=4.9151$ which means that the distribution is mesokurtic; (ii) greater than 3 when either $\alpha<2.4977$ or $\alpha>4.9151$ which means that the distribution is leptokurtic; (iii) smaller than 3 when $2.4977<\alpha<4.9151$ which means that the distribution is platykurtic. This analysis shows how the power parameter $\alpha$ improves $N(\beta)$, because the power transformation model can be used for data with a wide variety of distributional shapes.

5.4. Order Statistics. Consider $n$ independent and identical components whose lifetimes, say $X_{1}, X_{2}, \ldots, X_{n}$, follow $\mathrm{TN}(\alpha, \beta)$. The following theorem gives the limiting distributions of the lifetime of the series system $X_{1: n}=\min \left\{X_{1}, \ldots, X_{n}\right\}$ and of the parallel system $X_{n: n}=$ $\max \left\{X_{1}, \ldots, X_{n}\right\}$ consisting of these $n$ components.

Theorem 5. The limiting distributions of $X_{1: n}$ and $X_{n: n}$ are

$$
\begin{array}{r}
\lim _{n \rightarrow \infty} P\left\{\frac{X_{1: n}-a_{n}}{b_{n}} \leq x\right\}=1-e^{-x^{\alpha}}, \quad x>0, \\
\lim _{n \rightarrow \infty} P\left\{\frac{X_{n: n}-c_{n}}{d_{n}} \leq x\right\}=\exp \{-\exp (-x)\}, \\
x \in(-\infty, \infty),
\end{array}
$$

where $a_{n}=0, b_{n}=F^{-1}(1 / n), c_{n}=F^{-1}(1-1 / n), d_{n}=1 / n f\left(c_{n}\right)$ and $F(x)=1-S(x)$ is the cdf of $X$.
Proof. Using L'Hospital rule,

$$
\begin{aligned}
\lim _{t \rightarrow 0+} \frac{F(t x)}{F(t)} & =\lim _{t \rightarrow 0+} \frac{x f(t x)}{f(t)} \\
& =\lim _{t \rightarrow 0+} \frac{x^{\alpha}\left[\beta+\left(1+2 \beta(t x)^{\alpha}\right) e^{-\beta(t x)^{\alpha}}\right] e^{-\beta(t x)^{\alpha}}}{\left[\beta+\left(1+2 \beta t^{\alpha}\right) e^{-\beta t^{\alpha}}\right] e^{-\beta t^{\alpha}}} \\
& =x^{\alpha}
\end{aligned}
$$

Therefore, (19) follows by Theorem 8.3.6(ii) of Arnold et al. [4].

For the power transformation, we have

$$
\begin{aligned}
\lim _{x \rightarrow \infty} & \frac{d}{d x} \frac{1}{h(x)} \\
= & \lim _{x \rightarrow \infty}-\frac{(\alpha-1)\left(\beta+\left(1+\beta x^{\alpha}\right) e^{-\beta x^{\alpha}}\right)}{\alpha \beta x^{\alpha}\left(\beta+\left(1+2 \beta x^{\alpha}\right) e^{-\beta x^{\alpha}}\right)} \\
& +\lim _{x \rightarrow \infty} \frac{\beta x^{\alpha}}{1+2 \beta x^{\alpha}+\beta e^{\beta x^{\alpha}}} \\
& +\lim _{x \rightarrow \infty} \frac{\beta\left(2 \beta x^{\alpha}-1\right)+\left(\beta x^{\alpha}\left(1+2 \beta x^{\alpha}\right)-1\right) e^{-\beta x^{\alpha}}}{\left[\beta+\left(1+2 \beta x^{\alpha}\right) e^{-\beta x^{\alpha}}\right]^{2} e^{\beta x^{\alpha}}}=0 .
\end{aligned}
$$

Therefore, (20) follows by Theorem 8.3.3 of Arnold et al. [4].

The following Theorem gives the limiting distribution of the $i$ th order statistic of the $n$ lifetimes $X_{1}, X_{2}, \ldots, X_{n}$.

Theorem 6. The limiting distributions of $X_{i: n}, 1 \leq i \leq n$ are

$$
\lim _{n \rightarrow \infty} P\left\{\frac{X_{i: n}-a_{n}}{b_{n}} \leq x\right\}=1-\sum_{j=0}^{i-1} \frac{x^{j \alpha}}{j !} e^{-x^{\alpha}}, \quad x>0
$$

where $a_{n}=0, b_{n}=F^{-1}(1 / n)$.

Proof. It follows from Theorem 5 and (8.4.2) of Arnold et al. [4].

Theorem 5 means that $\left(X_{1: n}-a_{n}\right) / b_{n}$ and $\left(X_{n: n}-c_{n}\right) / d_{n}$ follow asymptotically $W(1, \alpha)$ and $\operatorname{Ext}(0,1)$, respectively, while Theorem 6 means that $\left(X_{i: n}-a_{n}\right) / b_{n}$ follows asymptotically $\operatorname{PG}(\alpha, i, 1)$.

\section{Maximum Likelihood Estimation}

Maximum likelihood estimation (MLE) is one of the most common methods for estimating the parameters of a statistical model. Assume that $n$ independent and identical items, whose lifetimes follow $\operatorname{TN}(\alpha, \beta)$, are put on a life test simultaneously. Let $x_{1}, x_{2}, \ldots, x_{n}$ be the failure times of 


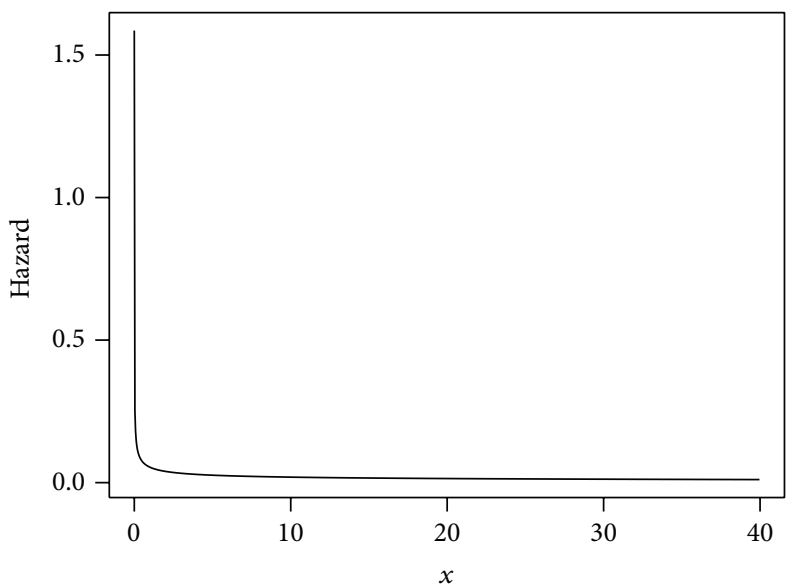

(a) $\alpha=0.5, \beta=0.1$

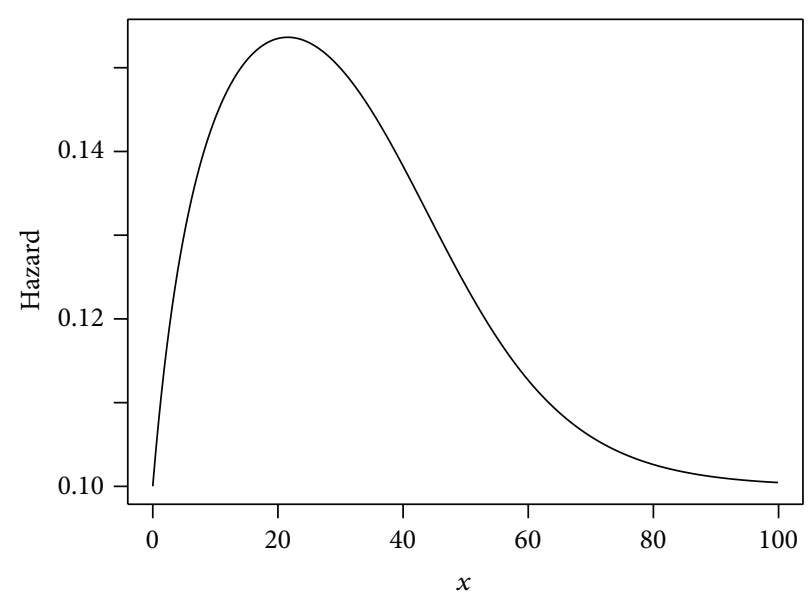

(c) $\alpha=1, \beta=0.1$

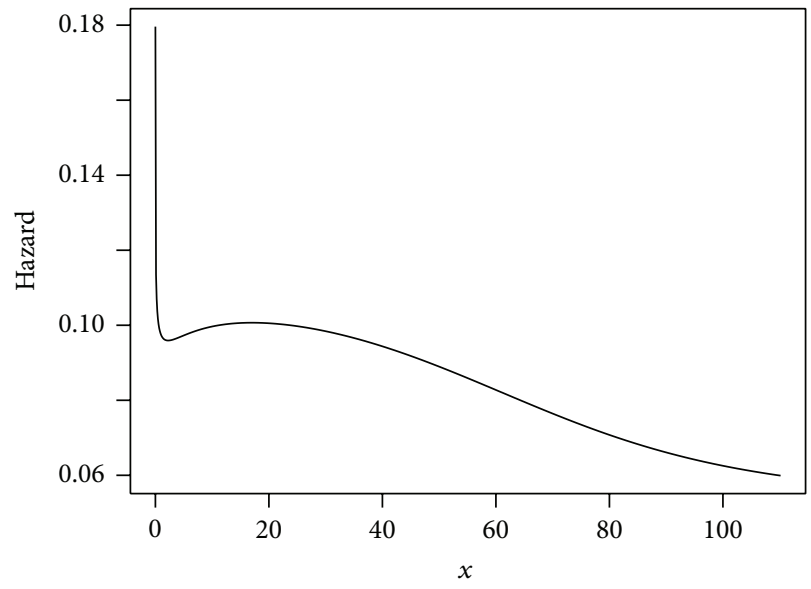

(b) $\alpha=0.9, \beta=0.1$

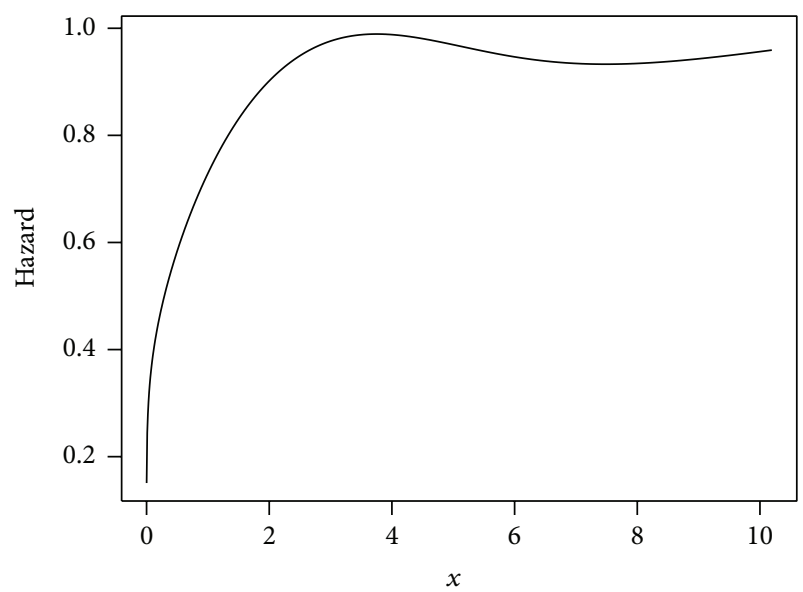

(d) $\alpha=1.2, \beta=0.5$

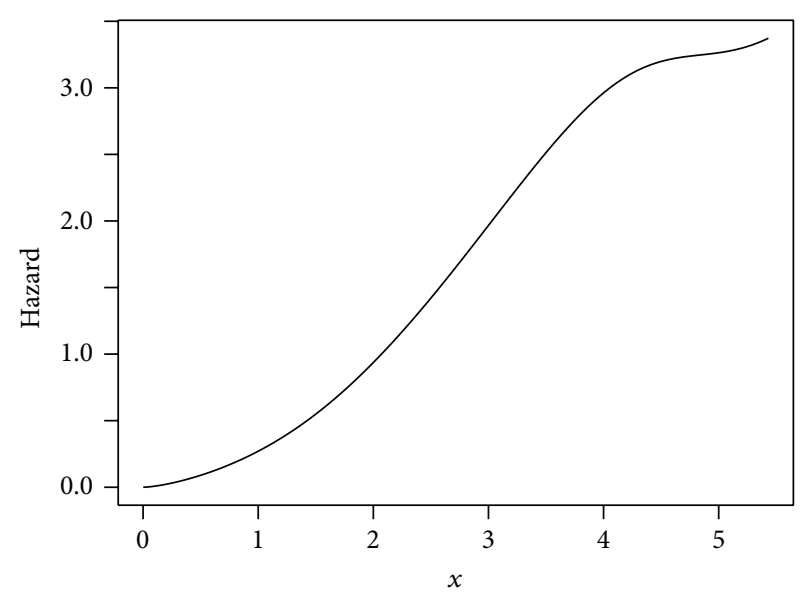

(e) $\alpha=2.5, \beta=0.1$

FIgURE 4: The hazard function of $\operatorname{TN}(\alpha, \beta)$ for some parameter values. 


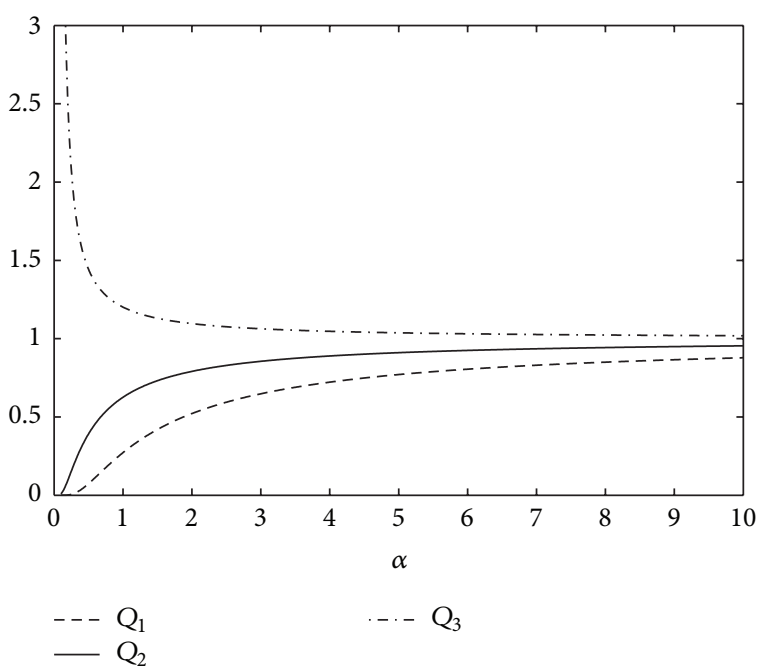

Figure 5: The quartiles of $\operatorname{TN}(\alpha, \beta)$ as functions of $\alpha$, when $\beta=1$.

the items and let $\mathbf{x}=\left(x_{1}, \ldots, x_{n}\right)$. The likelihood function for $(\alpha, \beta)$ is

$$
\begin{aligned}
L(\alpha, \beta ; \mathbf{x})= & \frac{\alpha^{n} \beta^{n}}{(1+\beta)^{n}}\left(\prod_{i=1}^{n} x_{i}\right)^{\alpha-1} e^{-\beta \sum_{i=1}^{n} x_{i}^{\alpha}} \\
& \times \prod_{i=1}^{n}\left[\beta+\left(1+2 \beta x_{i}^{\alpha}\right) e^{-\beta x_{i}^{\alpha}}\right] .
\end{aligned}
$$

The log-likelihood function is

$$
\begin{aligned}
\mathscr{L}(\alpha, \beta ; \mathbf{x})= & n \ln \alpha+n \ln \beta-n \ln (1+\beta) \\
& +(\alpha-1) \sum_{i=1}^{n} \ln x_{i}-\beta \sum_{i=1}^{n} x_{i}^{\alpha}+\sum_{i=1}^{n} \ln A_{i}(\alpha, \beta),
\end{aligned}
$$

where

$$
A_{i}(\alpha, \beta)=\beta+\left(1+2 \beta x_{i}^{\alpha}\right) e^{-\beta x_{i}^{\alpha}}, \quad i=1, \ldots, n .
$$

The first partial derivatives of $\mathscr{L}$, with respect to $\alpha$ and $\beta$, are

$$
\begin{gathered}
\mathscr{L}_{\alpha}=\frac{n}{\alpha}+\sum_{i=1}^{n} \ln x_{i}-\beta \sum_{i=1}^{n} x_{i}^{\alpha} \ln x_{i}+\sum_{i=1}^{n} \frac{A_{i, \alpha}(\alpha, \beta)}{A_{i}(\alpha, \beta)}, \\
\mathscr{L}_{\beta}=\frac{n}{\beta(1+\beta)}-\sum_{i=1}^{n} x_{i}^{\alpha}+\sum_{i=1}^{n} \frac{A_{i, \beta}(\alpha, \beta)}{A_{i}(\alpha, \beta)},
\end{gathered}
$$

where

$$
\begin{gathered}
A_{i, \alpha}(\alpha, \beta)=\frac{\partial A_{i}(\alpha, \beta)}{\partial \alpha}=\beta x_{i}^{\alpha}\left(1-2 \beta x_{i}^{\alpha}\right) \ln x_{i} e^{-\beta x_{i}^{\alpha}}, \\
A_{i, \beta}(\alpha, \beta)=\frac{\partial A_{i}(\alpha, \beta)}{\partial \beta}=1+\left(1-2 \beta x_{i}^{\alpha}\right) x_{i}^{\alpha} e^{-\beta x_{i}^{\alpha}} .
\end{gathered}
$$

The second partial derivatives of $\mathscr{L}$ are

$$
\begin{aligned}
\mathscr{L}_{\alpha, \alpha}= & -\frac{n}{\alpha^{2}}-\beta \sum_{i=1}^{n} x_{i}^{\alpha}\left(\ln x_{i}\right)^{2} \\
& +\sum_{i=1}^{n} \frac{A_{i}(\alpha, \beta) A_{i, \alpha^{2}}(\alpha, \beta)-\left[A_{i, \alpha}(\alpha, \beta)\right]^{2}}{\left[A_{i}(\alpha, \beta)\right]^{2}}, \\
\mathscr{L}_{\alpha, \beta}= & -\sum_{i=1}^{n} x_{i}^{\alpha} \ln x_{i} \\
& +\sum_{i=1}^{n} \frac{A_{i}(\alpha, \beta) A_{i, \alpha \beta}(\alpha, \beta)-A_{i, \alpha}(\alpha, \beta) A_{i, \beta}(\alpha, \beta)}{\left[A_{i}(\alpha, \beta)\right]^{2}}, \\
\mathscr{L}_{\beta, \beta}= & -\frac{n}{\beta^{2}}+\frac{n}{(1+\beta)^{2}} \quad \\
& +\sum_{i=1}^{n} \frac{A_{i}(\alpha, \beta) A_{i, \beta^{2}}(\alpha, \beta)-\left[A_{i, \beta}(\alpha, \beta)\right]^{2}}{\left[A_{i}(\alpha, \beta)\right]^{2}},
\end{aligned}
$$

where

$$
\begin{gathered}
A_{i, \alpha^{2}}(\alpha, \beta)=\beta x_{i}^{\alpha}\left(\ln x_{i}\right)^{2} e^{-\beta x_{i}^{\alpha}}\left(1-5 \beta x_{i}^{\alpha}+2 \beta^{2} x_{i}^{2 \alpha}\right), \\
A_{i, \alpha \beta}(\alpha, \beta)=x_{i}^{\alpha}\left(\ln x_{i}\right) e^{-\beta x_{i}^{\alpha}}\left(1-5 \beta x_{i}^{\alpha}+2 \beta^{2} x_{i}^{2 \alpha}\right), \\
A_{i, \beta^{2}}(\alpha, \beta)=-x_{i}^{2 \alpha} e^{-\beta x_{i}^{\alpha}}\left(3-2 \beta x_{i}^{\alpha}\right) .
\end{gathered}
$$

The information matrix is

$$
\mathfrak{F}(\alpha, \beta)=-\left[\begin{array}{ll}
\mathscr{L}_{\alpha, \alpha} & \mathscr{L}_{\alpha, \beta} \\
\mathscr{L}_{\alpha, \beta} & \mathscr{L}_{\beta, \beta}
\end{array}\right] .
$$

The MLE of $\alpha$ and $\beta$, say $\widehat{\alpha}$ and $\widehat{\beta}$, are the solution of the system of nonlinear equations obtained by setting $\mathscr{L}_{\alpha}=$ 0 and $\mathscr{L}_{\beta}=0$ such that the $\mathfrak{F}(\widehat{\alpha}, \widehat{\beta})$ is positive definite. This system has no analytic solution, so numerical methods, such as the Newton-Raphson method, Burden and Faires [5], should be used.

Large-Sample Intervals. The MLE of the parameters $\alpha$ and $\beta$ are asymptotically normally distributed with means equal to the true values of $\alpha$ and $\beta$ and variances given by the inverse of the information matrix. In particular,

$$
\left(\begin{array}{l}
\widehat{\alpha} \\
\widehat{\beta}
\end{array}\right) \sim N_{2}\left(\left(\begin{array}{l}
\alpha \\
\beta
\end{array}\right), \widehat{\Im}^{-1}\right),
$$

where $\widehat{\mathscr{F}}^{-1}$ is the inverse of $\mathfrak{F}(\widehat{\alpha}, \widehat{\beta})$, with main diagonal elements $\widehat{\mathfrak{F}}^{11}$ and $\widehat{\mathfrak{F}}^{22}$ given by

$$
\widehat{\mathfrak{F}}^{11}=\frac{\widehat{\mathfrak{F}}_{22}}{\widehat{\mathfrak{\mho}}_{11} \widehat{\mho}_{22}-\widehat{\mathfrak{F}}_{12}^{2}}, \quad \widehat{\mathfrak{F}}^{22}=\frac{\widehat{\Im}_{11}}{\widehat{\mathfrak{F}}_{11} \widehat{\Im}_{22}-\widehat{\mathfrak{F}}_{12}^{2}} .
$$

Using (32), large-sample $(1-9) 100 \%$ confidence intervals for $\alpha$ and $\beta$ are

$$
\widehat{\alpha} \pm Z_{\vartheta / 2} \sqrt{\widehat{\widetilde{F}}^{11}}, \quad \widehat{\beta} \pm Z_{\vartheta / 2} \sqrt{\widehat{\widetilde{F}}^{22}},
$$

where $Z_{9 / 2}$ is the upper $1009 / 2$ quantile of the standard normal distribution. 

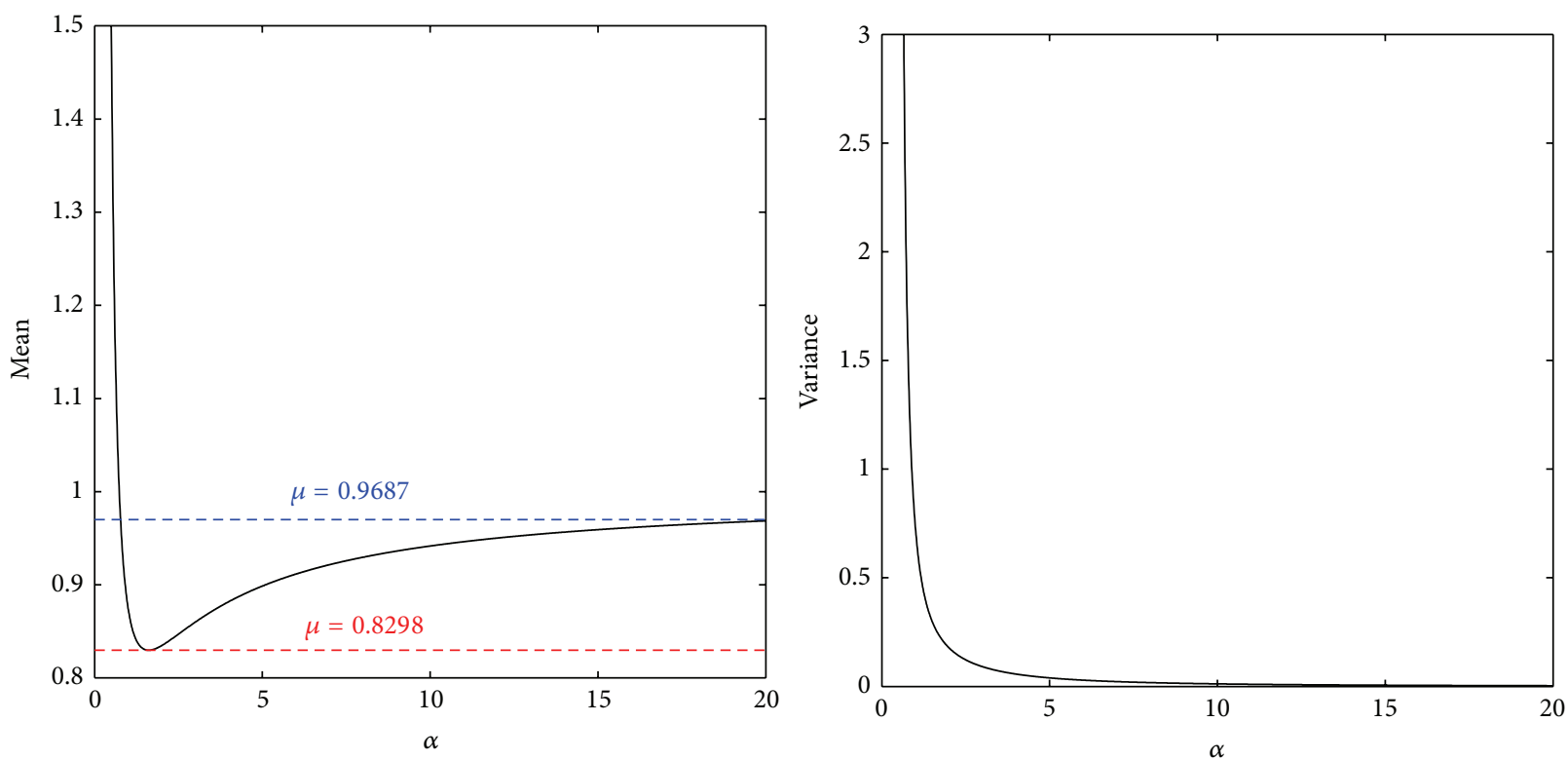

FIGURE 6: The mean and variance of $\operatorname{TN}(\alpha, \beta)$ as functions of $\alpha$, when $\beta=1$.
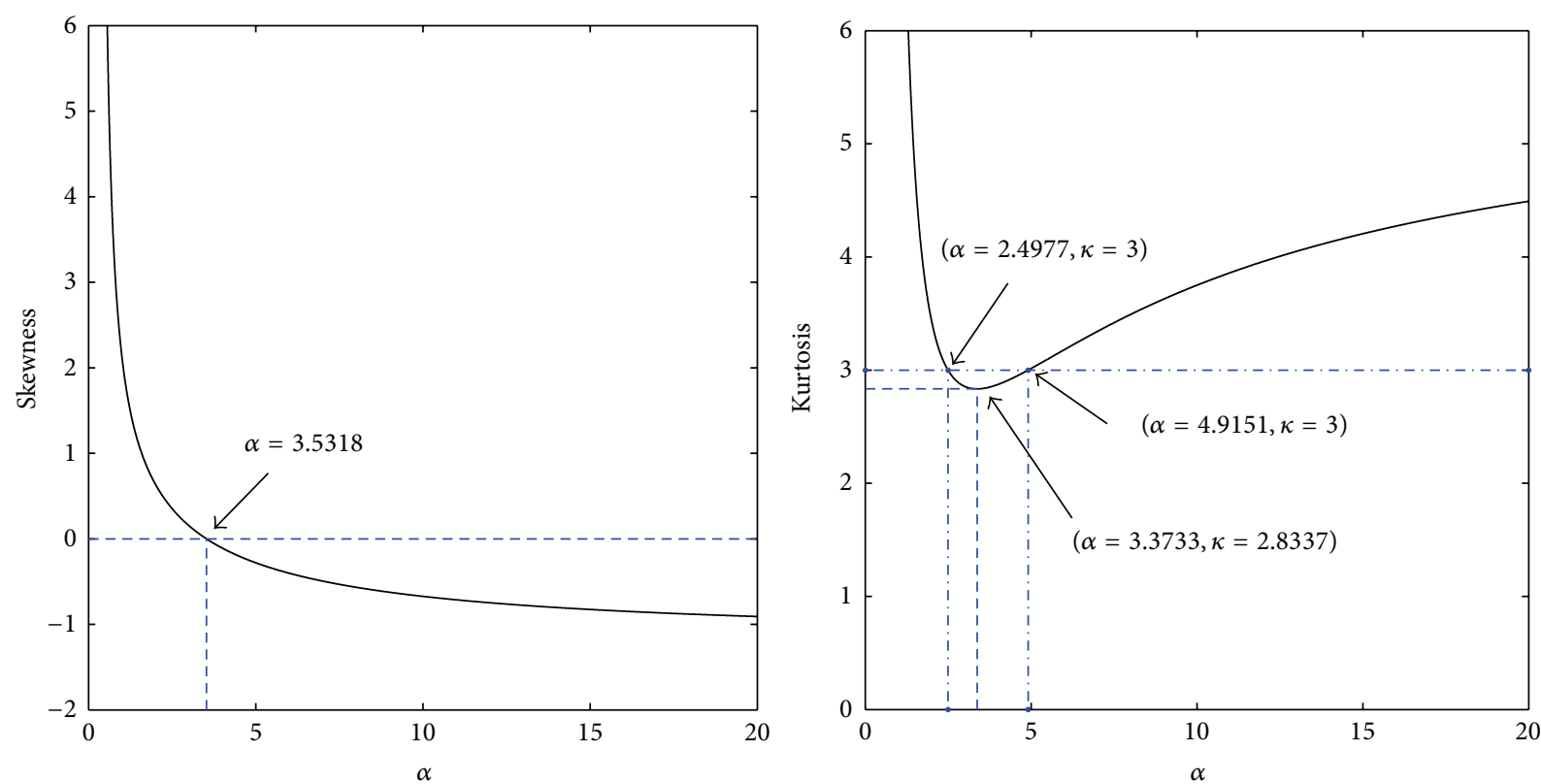

Figure 7: The skewness and kurtosis of $\operatorname{TN}(\alpha, \beta)$ as functions of $\alpha$, when $\beta=1$.

\section{Applications}

In this section, we analyze four data sets to illustrate the applicability of the two new distributions proposed in this paper. The first data consists of 61 observed recidivism failure times (in days) of individuals released directly from correctional institutions to parole in the District of Columbia, Columbia, USA [6]. The second data set consists of 43 active repair times (in hours) for an airborne communication transceiver [7]. The third data set consists of 57 times (in thousands of operating hours) of unscheduled maintenance actions for the number 4 diesel engine of the U.S.S. Grampus, up to 16 thousand hours of operation [8]. The forth data set consists of the tensile strength (measured in GPa) of 69 carbon fibers tested under tension at gauge lengths of $20 \mathrm{~mm}$ [9].

We will refer to these data sets as failure times, repair times, maintenance actions, and tensile strength data, respectively. For each data set, we fit the proposed two distributions as well as Lindley and power Lindley distributions. For 

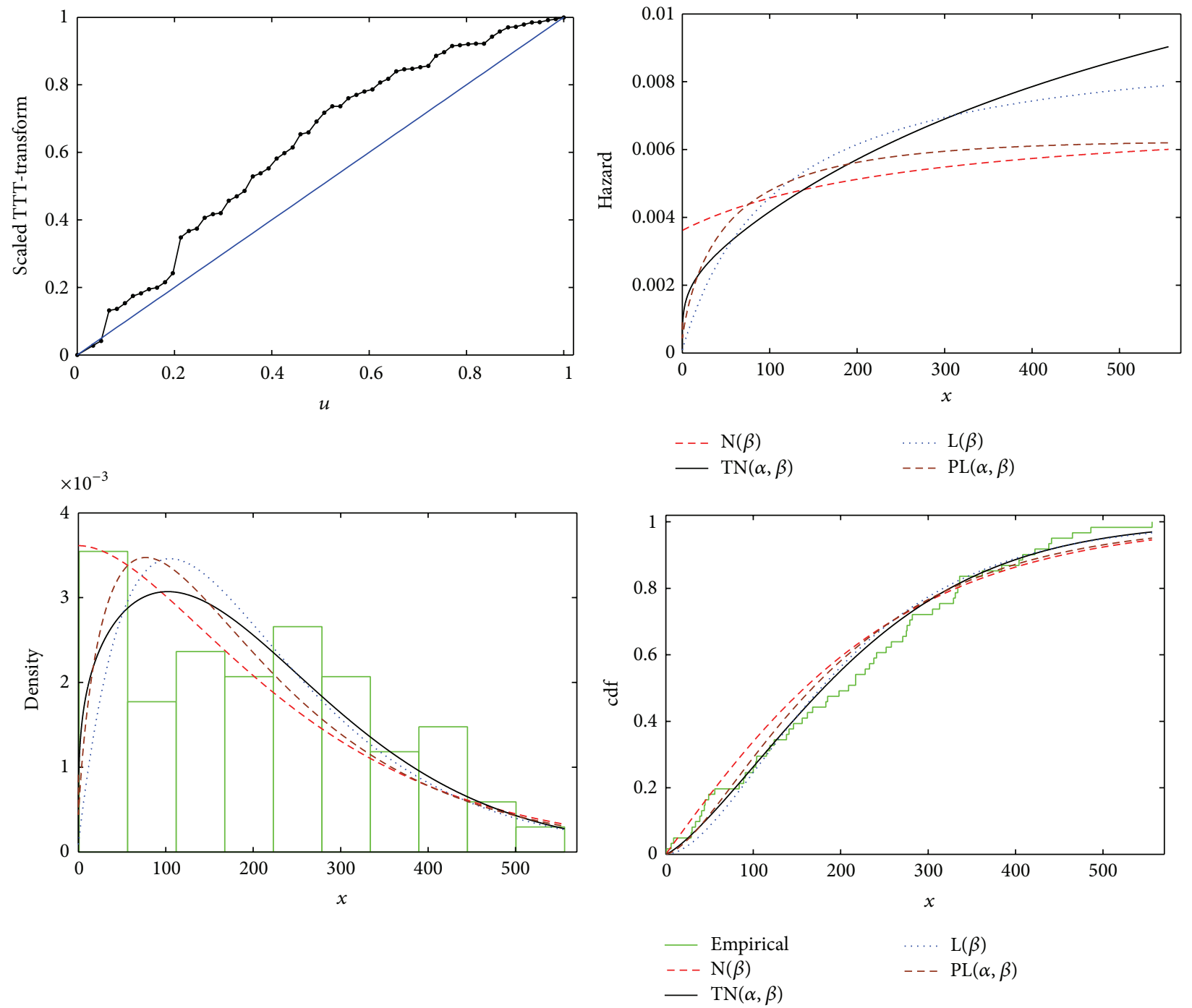

FIGURE 8: The TTT-transform, fitted hazard, pdf, and cdf of the failure times data.

the sake of comparison, we apply goodness-of-fit tests to verify which distribution better fits these data sets. We consider the well-known Kolmogorov-Smirnov (K-S) statistic, the Cramér-von Mises (C-M), and Anderson-Darling (A-D) statistics [10]. Furthermore, we consider the Akaike information criterion AIC $=-2 \widehat{\mathscr{L}}+2 q$, where $\widehat{\mathscr{L}}$ is the loglikelihood function at the MLE of the parameters and $q$ is the number of model parameters. Table 1 shows the MLE of the parameters of each model, the corresponding maximum loglikelihood value, and the AIC for the four data sets. Table 2 presents the results of the goodness of fit tests for the four data sets using each model.

For every data set, we plotted (1) the scaled total time on test transform (TTT-transform) plot which gives qualitative information about the hazard rate shape [11]; (2) the hazard functions for the four fitted models; (3) the empirical and fitted density and distribution functions. Figures 8, 9, 10, and 11 show the four plots for the four data sets $1-4$, respectively. The scaled TTT-transform plots show that the repair data set has a unimodal hazard, while the rest of data sets have increasing hazards.

The inverse of information matrix at the MLE using the four data sets are listed below.

Failure times:

$$
\widehat{\mathfrak{F}}^{-1}=\left[\begin{array}{cc}
1.717231 \times 10^{-3} & -1.024661 \times 10^{-5} \\
-1.024661 \times 10^{-5} & 5.019728 \times 10^{-7}
\end{array}\right] .
$$

Active repair:

$$
\widehat{\Im}^{-1}=\left[\begin{array}{cc}
0.00901512 & -0.00442645 \\
-0.00442645 & 0.00430030
\end{array}\right]
$$

Maintenance actions:

$$
\widehat{\mathfrak{F}}^{-1}=\left[\begin{array}{cc}
0.046064730 & -1.998013 \times 10^{-3} \\
-1.998013 \times 10^{-3} & 9.07815 \times 10^{-5}
\end{array}\right] .
$$

Tensile strength:

$$
\widehat{\mathfrak{F}}^{-1}=\left[\begin{array}{cc}
0.521162636 & -3.846724 \times 10^{-3} \\
-3.846724 \times 10^{-3} & 2.892066 \times 10^{-5}
\end{array}\right] .
$$



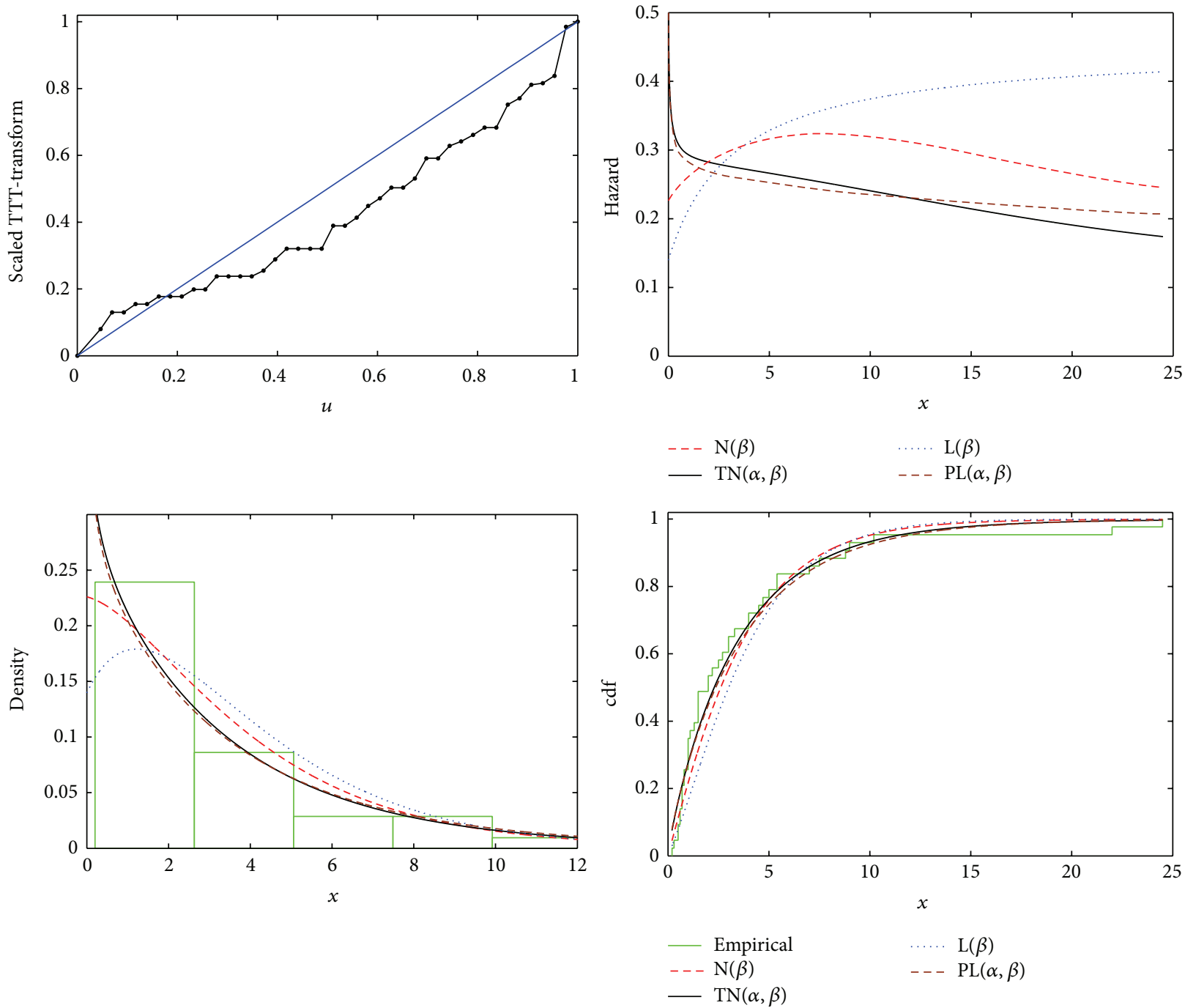

Figure 9: The TTT-transform, hazard, pdf, and cdf of the active repair times data.

TABLE 1: Parameter estimates, maximum log-likelihood, and AIC for the four data sets.

\begin{tabular}{|c|c|c|c|c|}
\hline Data & Model & Parameter estimates & $\widehat{\mathscr{L}}$ & AIC \\
\hline \multirow{4}{*}{ Failure times } & $\mathrm{L}(\beta)$ & $\widehat{\beta}=9.403 \times 10^{-3}$ & -386.894 & 775.789 \\
\hline & $\operatorname{PL}(\alpha, \beta)$ & $\widehat{\alpha}=0.8778, \widehat{\beta}=0.0185$ & -385.958 & 775.916 \\
\hline & $\mathrm{N}(\beta)$ & $\widehat{\beta}=3.61354 \times 10^{-3}$ & -384.872 & 771.743 \\
\hline & $\mathrm{TN}(\alpha, \beta)$ & $\widehat{\alpha}=1.2551, \widehat{\beta}=8.442 \times 10^{-4}$ & -382.904 & 769.808 \\
\hline \multirow{4}{*}{ Repair times } & $\mathrm{L}(\beta)$ & $\widehat{\beta}=0.449891$ & -104.433 & 210.865 \\
\hline & $\operatorname{PL}(\alpha, \beta)$ & $\widehat{\alpha}=0.762291, \widehat{\beta}=0.652788$ & -99.974 & 203.947 \\
\hline & $\mathrm{N}(\beta)$ & $\widehat{\beta}=0.226013$ & -100.821 & 203.642 \\
\hline & $\operatorname{TN}(\alpha, \beta)$ & $\widehat{\alpha}=0.843574, \widehat{\beta}=0.295802$ & -99.572 & 203.144 \\
\hline \multirow{4}{*}{ Maintenance actions } & $\mathrm{L}(\beta)$ & $\widehat{\beta}=0.217325$ & -170.317 & 342.634 \\
\hline & $\operatorname{PL}(\alpha, \beta)$ & $\widehat{\alpha}=1.28229, \widehat{\beta}=0.117289$ & -167.089 & 338.178 \\
\hline & $\mathrm{N}(\beta)$ & $\widehat{\beta}=0.0936263$ & -174.636 & 351.271 \\
\hline & $\mathrm{TN}(\alpha, \beta)$ & $\widehat{\alpha}=1.69539, \widehat{\beta}=0.0172624$ & -165.584 & 335.167 \\
\hline \multirow{4}{*}{ Tensile strength } & $\mathrm{L}(\beta)$ & $\widehat{\beta}=0.6545$ & -119.190 & 240.381 \\
\hline & $\operatorname{PL}(\alpha, \beta)$ & $\widehat{\alpha}=3.8678, \widehat{\beta}=0.0497$ & -49.060 & 102.119 \\
\hline & $\mathrm{N}(\beta)$ & $\widehat{\beta}=0.3334$ & -124.618 & 251.235 \\
\hline & $\mathrm{TN}(\alpha, \beta)$ & $\widehat{\alpha}=4.8853, \widehat{\beta}=0.0067$ & -49.976 & 103.952 \\
\hline
\end{tabular}



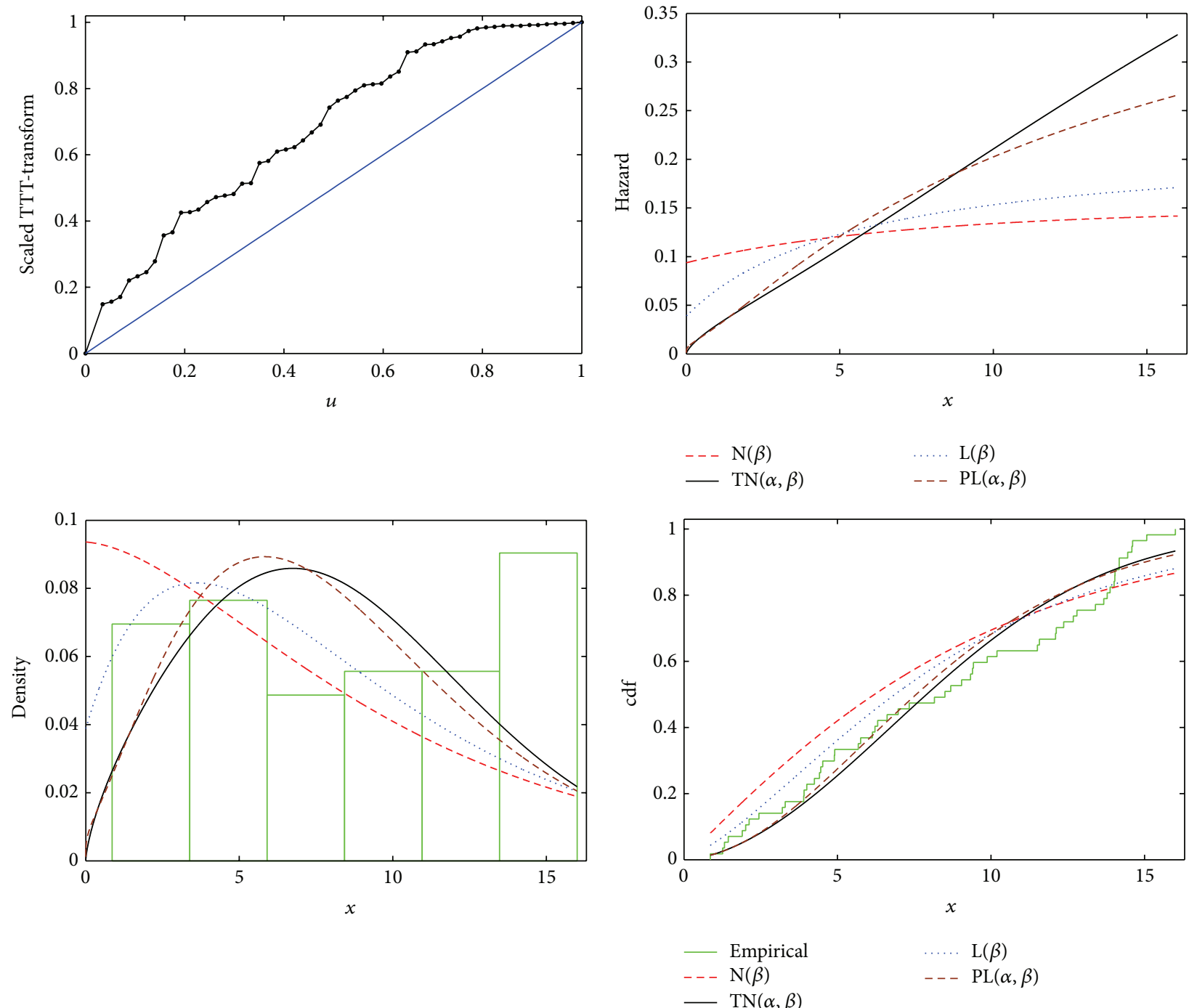

FIGURE 10: The TTT-transform, hazard, pdf, and cdf of the maintenance actions data.

TABle 2: Statistics K-S (P value), C-M, and A-D for the four data sets.

\begin{tabular}{|c|c|c|c|c|}
\hline Data & Model & K-S ( $P$ value $)$ & C-M & A-D \\
\hline \multirow{4}{*}{ Failure times } & $\mathrm{L}(\beta)$ & $0.099087(0.55362)$ & 0.1456121 & 0.907295 \\
\hline & $\operatorname{PL}(\alpha, \beta)$ & $0.094044(0.61929)$ & 0.1632937 & 1.013002 \\
\hline & $\mathrm{N}(\beta)$ & $0.103937(0.49274)$ & 0.1175828 & 0.741661 \\
\hline & $\mathrm{TN}(\alpha, \beta)$ & $0.069538(0.90959)$ & 0.0847141 & 0.542494 \\
\hline \multirow{4}{*}{ Repair times } & $\mathrm{L}(\beta)$ & $0.234632(0.01449)$ & 0.1810547 & 1.210262 \\
\hline & $\operatorname{PL}(\alpha, \beta)$ & $0.120462(0.52160)$ & 0.1330721 & 0.895534 \\
\hline & $\mathrm{N}(\beta)$ & $0.174114(0.13065)$ & 0.1506954 & 0.993861 \\
\hline & $\operatorname{TN}(\alpha, \beta)$ & $0.114174(0.58940)$ & 0.1268951 & 0.840214 \\
\hline \multirow{4}{*}{ Maintenance actions } & $\mathrm{L}(\beta)$ & $0.122002(0.33663)$ & 0.187688 & 1.305582 \\
\hline & $\operatorname{PL}(\alpha, \beta)$ & $0.119731(0.35863)$ & 0.178752 & 1.244465 \\
\hline & $\mathrm{N}(\beta)$ & $0.146367(0.15728)$ & 0.186369 & 1.298771 \\
\hline & $\mathrm{TN}(\alpha, \beta)$ & $0.111731(0.44316)$ & 0.160603 & 1.120745 \\
\hline \multirow{4}{*}{ Tensile strength } & $\mathrm{L}(\beta)$ & $0.386637\left(1.17899 \times 10^{-9}\right)$ & 0.0403019 & 0.3025705 \\
\hline & $\operatorname{PL}(\alpha, \beta)$ & $0.044275(0.99840)$ & 0.0181847 & 0.1619866 \\
\hline & $\mathrm{N}(\beta)$ & $0.415150\left(4.57128 \times 10^{-11}\right)$ & 0.0432435 & 0.3221342 \\
\hline & $\mathrm{TN}(\alpha, \beta)$ & $0.056805(0.96997)$ & 0.0403920 & 0.3132999 \\
\hline
\end{tabular}



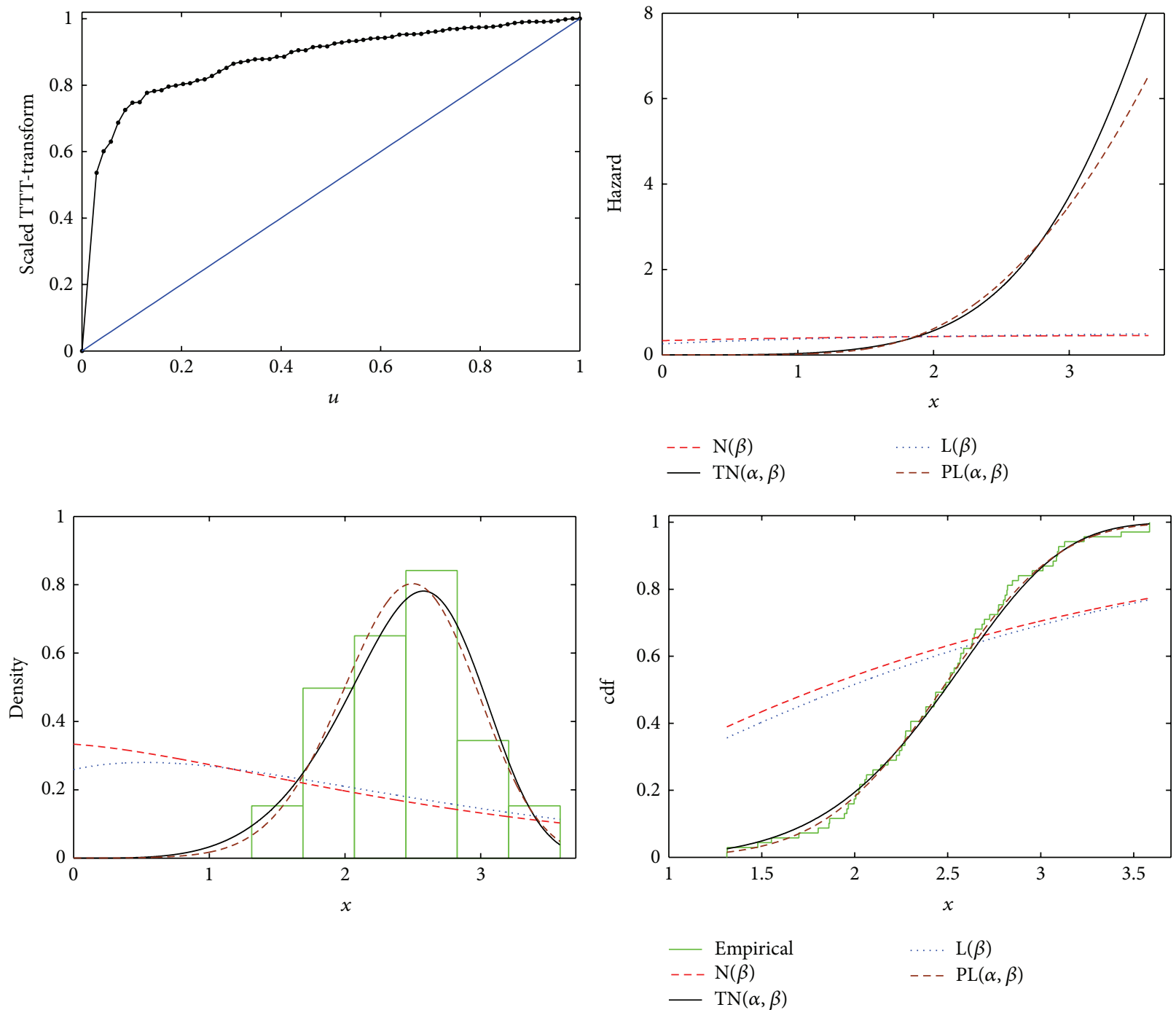

FIGURE 11: The TTT-transform, hazard, pdf, and cdf of the tensile strength data.

TABLE 3: The LRT and $P$ value for the four data sets.

\begin{tabular}{lcccc}
\hline & $\begin{array}{c}\text { Failure } \\
\text { times }\end{array}$ & $\begin{array}{c}\text { Repair } \\
\text { times }\end{array}$ & $\begin{array}{c}\text { Maintenance } \\
\text { actions }\end{array}$ & $\begin{array}{c}\text { Tensile } \\
\text { strength }\end{array}$ \\
\hline$\Lambda$ & 3.936 & 2.498 & 18.104 & 149.284 \\
$P$ value & 0.0473 & 0.1140 & $2.0916 \times 10^{-5}$ & 0 \\
\hline
\end{tabular}

For the first three data sets, $\operatorname{TN}(\alpha, \beta)$ model has the smallest value of the Kolmogorov-Smirnov (largest $P$ value), the Cramér-von Mises, and Anderson-Darling goodness-offit tests statistics which indicate that the best fit is provided by the TN model for these data sets. For the forth data set, the power Lindley model provides the best fit in the sense of having the smallest test statistics. For all data sets, $\operatorname{TN}(\alpha, \beta)$ is a better fit than $N(\beta)$. For the first two data sets, $N(\beta)$ is a better fit than both $L(\beta)$ and $\operatorname{PL}(\alpha, \beta)$ while it is the worst fit for the last two data sets. The AIC statistic is the lowest for $\mathrm{TN}(\alpha, \beta)$ for all data sets except for tensile strength where it is slightly higher.

Further, for testing $N(\beta)$ as a submodel of the $\operatorname{TN}(\alpha, \beta)$, we use the likelihood ratio test statistic (LRT) to check if the fit using the $\mathrm{TN}(\alpha, \beta)$ is statistically superior to a fit using the $N(\beta)$ for each data set. The LRT for testing $H_{0}$ : $\alpha=1$ against $H_{1}: \alpha \neq 1$ is $\Lambda=2\left(\widehat{\mathscr{L}}_{H_{1}}-\widehat{\mathscr{L}}_{H_{0}}\right)$, where $\widehat{\mathscr{L}}_{H_{1}}$ and $\widehat{\mathscr{L}}_{H_{0}}$ are the maximum log-likelihood values under $H_{1}$ and $H_{0}$, respectively. Under $H_{0}, \Lambda \stackrel{d}{\rightarrow} \chi_{1}^{2}$. The LRT rejects $H_{0}$ if $\Lambda>\chi_{1, \gamma}^{2}$, where $\chi_{1, \gamma}^{2}$ denotes the upper $100 \gamma \%$ point of chi-square distribution with 1 degree of freedom. Table 3 lists the values of the LRT and the corresponding $P$ value for the four data sets. Based on the $P$ values, the $N(\beta)$ is not rejected against the $\operatorname{TN}(\alpha, \beta)$ to fit the repair times data set, while it is rejected, at any level of 
TABLE 4: MSE, coverage probability, and average width.

\begin{tabular}{|c|c|c|c|c|c|c|c|c|}
\hline$\alpha$ & $\beta$ & $n$ & $\mathrm{MSE}_{\alpha}$ & $\mathrm{MSE}_{\beta}$ & $\mathrm{CP}_{\alpha}$ & $\mathrm{AW}_{\alpha}$ & $\mathrm{CP}_{\beta}$ & $\mathrm{AW}_{\beta}$ \\
\hline \multirow{4}{*}{0.5} & \multirow{4}{*}{1} & 25 & 0.008889 & 0.0639280 & 0.9550 & 0.335546 & 0.9426 & 0.888835 \\
\hline & & 50 & 0.003720 & 0.0250429 & 0.9553 & 0.229186 & 0.9541 & 0.608075 \\
\hline & & 75 & 0.002295 & 0.0158072 & 0.9558 & 0.184697 & 0.9527 & 0.491875 \\
\hline & & 100 & 0.001690 & 0.0120432 & 0.9547 & 0.159216 & 0.9528 & 0.425521 \\
\hline \multirow{4}{*}{1.0} & \multirow{4}{*}{1.0} & 25 & 0.035209 & 0.0603732 & 0.9552 & 0.669487 & 0.9492 & 0.884671 \\
\hline & & 50 & 0.015100 & 0.0259297 & 0.9530 & 0.458663 & 0.9504 & 0.60922 \\
\hline & & 75 & 0.009433 & 0.0165418 & 0.9495 & 0.369965 & 0.9516 & 0.493743 \\
\hline & & 100 & 0.006956 & 0.0121903 & 0.9517 & 0.318365 & 0.9498 & 0.425539 \\
\hline \multirow{4}{*}{2.0} & \multirow{4}{*}{1.0} & 25 & 0.144086 & 0.0609798 & 0.9518 & 1.342590 & 0.9435 & 0.883723 \\
\hline & & 50 & 0.061349 & 0.0260667 & 0.9472 & 0.916073 & 0.9458 & 0.608569 \\
\hline & & 75 & 0.037822 & 0.0163597 & 0.9526 & 0.740067 & 0.9508 & 0.492714 \\
\hline & & 100 & 0.028013 & 0.0119553 & 0.9492 & 0.638045 & 0.9510 & 0.424817 \\
\hline \multirow{4}{*}{1.3} & \multirow{4}{*}{0.5} & 25 & 0.058995 & 0.0173859 & 0.9532 & 0.866467 & 0.9216 & 0.481574 \\
\hline & & 50 & 0.024938 & 0.0081550 & 0.9535 & 0.594661 & 0.9348 & 0.339487 \\
\hline & & 75 & 0.015840 & 0.0052273 & 0.9557 & 0.480683 & 0.9400 & 0.277333 \\
\hline & & 100 & 0.011542 & 0.0037797 & 0.9551 & 0.414303 & 0.9451 & 0.239666 \\
\hline \multirow{4}{*}{0.5} & \multirow{4}{*}{1.2} & 25 & 0.009273 & 0.0972282 & 0.9480 & 0.335704 & 0.9499 & 1.084880 \\
\hline & & 50 & 0.003832 & 0.0381008 & 0.9485 & 0.229018 & 0.9494 & 0.733431 \\
\hline & & 75 & 0.002355 & 0.0239632 & 0.9511 & 0.184765 & 0.9565 & 0.593873 \\
\hline & & 100 & 0.001787 & 0.0173496 & 0.9485 & 0.159225 & 0.9531 & 0.510691 \\
\hline \multirow{4}{*}{1.0} & \multirow{4}{*}{1.2} & 25 & 0.036640 & 0.0898864 & 0.9502 & 0.670473 & 0.9556 & 1.075180 \\
\hline & & 50 & 0.015133 & 0.0380503 & 0.9525 & 0.457310 & 0.9526 & 0.735221 \\
\hline & & 75 & 0.009416 & 0.0240831 & 0.9525 & 0.369850 & 0.9547 & 0.594754 \\
\hline & & 100 & 0.007055 & 0.0174628 & 0.9486 & 0.318305 & 0.9523 & 0.510473 \\
\hline \multirow{4}{*}{2.0} & \multirow{4}{*}{1.2} & 25 & 0.148128 & 0.0929780 & 0.9515 & 1.344300 & 0.9493 & 1.076180 \\
\hline & & 50 & 0.061533 & 0.0386808 & 0.9522 & 0.916556 & 0.9522 & 0.734676 \\
\hline & & 75 & 0.038607 & 0.0248240 & 0.9495 & 0.738584 & 0.9492 & 0.593036 \\
\hline & & 100 & 0.028413 & 0.0172320 & 0.9474 & 0.636959 & 0.9541 & 0.509967 \\
\hline \multirow{4}{*}{1.3} & \multirow{4}{*}{1.5} & 25 & 0.063330 & 0.1753970 & 0.9534 & 0.875085 & 0.9601 & 1.415120 \\
\hline & & 50 & 0.025851 & 0.0651604 & 0.9519 & 0.596480 & 0.9537 & 0.945666 \\
\hline & & 75 & 0.016517 & 0.0404539 & 0.9509 & 0.481512 & 0.9536 & 0.759421 \\
\hline & & 100 & 0.011529 & 0.0292367 & 0.9548 & 0.414153 & 0.9552 & 0.652792 \\
\hline \multirow{4}{*}{0.9} & \multirow{4}{*}{1.2} & 25 & 0.029932 & 0.0929842 & 0.9499 & 0.604276 & 0.9495 & 1.074170 \\
\hline & & 50 & 0.012327 & 0.0389142 & 0.9501 & 0.411230 & 0.9509 & 0.734209 \\
\hline & & 75 & 0.007675 & 0.0238699 & 0.9504 & 0.332339 & 0.9531 & 0.594194 \\
\hline & & 100 & 0.005652 & 0.0174031 & 0.9531 & 0.286597 & 0.9505 & 0.510094 \\
\hline
\end{tabular}

significance greater than or equal to 0.0473 , to fit the other three data sets.

\section{Simulation Study}

We used a simulation study to investigate the performance of the accuracy of point and interval estimates of the two parameters of $\operatorname{TN}(\alpha, \beta)$. The following steps are as follows:

(1) Specify the values of the parameters $\alpha$ and $\beta$;

(2) Specify the sample size $n$;

(3) Use Algorithm 2 and the transformation to generate a random sample with size $n$ from $\operatorname{TN}(\alpha, \beta)$. (a) Calculate the MLE of the two parameters and the inverse of the Fisher matrix;

(b) Calculate the squared deviation of the MLE from the exact value of each parameter;

(c) Calculate a 95\% CI for each parameter;

(4) Repeat steps 2-3, $N$ times;

(5) Calculate the mean squared error (MSE), the average of the confidence interval widths, and the coverage probability for each parameter. The MSE associated with the MLE of the parameter $\vartheta, \mathrm{MSE}_{\vartheta}$, is

$$
\mathrm{MSE}_{\vartheta}=\frac{1}{N} \sum_{i=1}^{N}\left(\widehat{\vartheta}_{i}-\vartheta\right)^{2},
$$


where $\widehat{\vartheta}_{i}$ is the MLE of $\vartheta$ using the $i$ th sample, $i=$ $1,2, \ldots, N$, and $\vartheta=\alpha, \beta$. Coverage probability is the proportion of the $N$ simulated confidence intervals which include the true parameter $\vartheta$.

The simulation study is used when $N=10,000$, the sample sizes are $25,50,75$, and 100 , and the parameter values $(\alpha, \beta)=$ $(0.5,1),(1,1),(2,1),(1.3,0.5),(0.5,1.2),(1,1.2),(2,1.2)$,

$(1.3,1.5)$, and $(0.9,1.2)$. Some of the selected values of $(\alpha, \beta)$ give decreasing, unimodal, increasing, and increasing-decreasing-increasing hazard shapes, respectively, as shown in Figure 3. Table 4 presents the MSE, coverage probability $\left(\mathrm{CP}_{\vartheta}\right)$, and average width $(\mathrm{AW})$ of $95 \%$ confidence intervals of each parameter. As it was expected, this table shows that the MSEs of the estimates decrease as the sample size increases, that the coverage probabilities are very close to the nominal level of $95 \%$, and that the average widths decrease as the sample size increases.

\section{Conclusion}

In this paper, we have proposed new one-parameter and two-parameter distributions, called the $N(\beta)$ and $\operatorname{TN}(\alpha, \beta)$, respectively. The $\mathrm{TN}(\alpha, \beta)$ was obtained by using a power transformation of the $N(\beta)$ distributed variable. The $\mathrm{TN}(\alpha, \beta)$ provides more flexibility than the $N(\beta)$ in terms of the shape of the density and hazard rate functions as well as its skewness and kurtosis. We derived the maximum likelihood estimates of the parameters and their variancecovariance matrix. We proposed different algorithms to generate samples from the two proposed distributions. Applications of the two proposed distributions to real data sets show better fits than Lindley and power Lindley distributions. Finally, we examined the accuracy of the maximum likelihood estimators of the $\mathrm{TN}(\alpha, \beta)$ parameters as well as the coverage probability and average width of the confidence intervals for the parameters using simulation.

\section{Conflict of Interests}

The authors declare that there is no conflict of interests regarding the publication of this paper.

\section{References}

[1] D. V. Lindley, "Fiducial distributions and Bayes' theorem," Journal of the Royal Statistical Society B. Methodological, vol. 20, pp. 102-107, 1958.

[2] M. E. Ghitany, B. Atieh, and S. Nadarajah, "Lindley distribution and its application," Mathematics and Computers in Simulation, vol. 78, no. 4, pp. 493-506, 2008.

[3] M. E. Ghitany, D. K. Al-Mutairi, N. Balakrishnan, and L. J. AlEnezi, "Power Lindley distribution and associated inference," Computational Statistics \& Data Analysis, vol. 64, pp. 20-33, 2013.

[4] B. C. Arnold, N. Balakrishnan, and H. N. Nagaraja, A First Course in Order Statistics, John Wiley \& Sons, New York, NY, USA, 1992.

[5] L. Burden and J. D. Faires, Numerical Analysis, Brooks/Cole, Cengage Learing, Stamford, Conn, USA, 9th edition, 2011.

[6] S. Stollmack and C. M. Harris, "Failure-rate analysis applied to recidivism data," Operations Research, vol. 22, no. 6, pp. 1192$1205,1974$.

[7] B. Jørgensen, Statistical Properties of the Generalized Inverse Gaussian Distribution, vol. 9, Springer, Heidelberg, Germany, 1982.

[8] W. O. Meeker and L. A. Escobar, Statistical Methods for Reliability Data, John Wiley \& Sons, New York, NY, USA, 1998.

[9] M. G. Bader and A. M. Priest, "Statistical aspects of fiber and bundle strength in hybrid composites," in Progress In Science and EngIneerIng Composites, T. Hayashi, K. Kawata, and S. Umekawa, Eds., pp. 1129-1136, ICCM-IV, Tokyo, Japan, 1982.

[10] G. Chen and N. Balakrishnan, "A general purpose approximate goodnessoffit test," Journal of Quality Technology, vol. 27, pp. 154-161, 1995.

[11] M. V. Aarset, "How to identify a bathtub hazard rate," IEEE Transactions on Reliability, vol. R-36, no. 1, pp. 106-108, 1987.

\section{Notation}

$$
\begin{aligned}
& \text { pdf: } \quad \text { Probability density function } \\
& \text { cdf: Cumulative distribution function } \\
& \text { mgf: } \quad \text { Moment generating function } \\
& U(0,1): \quad \text { Uniform distribution on }(0,1) \\
& \operatorname{Exp}(\beta): \quad \text { Exponential distribution with mean } 1 / \beta \\
& W(\nu, \beta): \quad \text { Weibull distribution with pdf } \\
& f(x)=\nu \beta x^{\nu-1} e^{-\beta x^{\nu}} \\
& G(\nu, \beta): \quad \text { Gamma distribution with pdf } \\
& f(x)=\left(\beta^{\nu} / \Gamma(\nu)\right) x^{\nu-1} e^{-\beta x} \\
& f(x)=\left(\alpha \beta^{\nu} / \Gamma(\nu)\right) x^{\alpha \nu-1} e^{-\beta x^{\alpha}} \\
& \operatorname{PL}(\alpha, \beta) \text { : } \quad \text { Power Lindley distribution with pdf } \\
& f(x)=\left(\alpha \beta^{2} /(1+\beta)\right) x^{\alpha-1}\left(1+x^{\alpha}\right) e^{-\beta x^{\alpha}} \\
& \operatorname{Ext}(\alpha, \beta) \text { : Extreme-value distribution with pdf } \\
& f(x)=(1 / \beta) e^{-(x-\alpha) / \beta-\exp \{-(x-\alpha) / \beta\}} .
\end{aligned}
$$




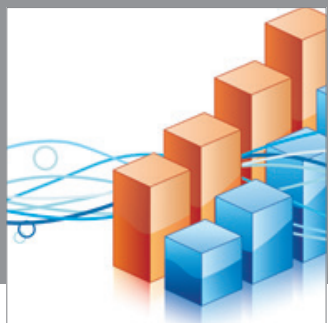

Advances in

Operations Research

mansans

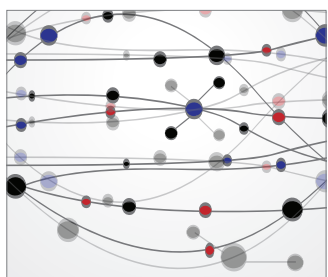

The Scientific World Journal
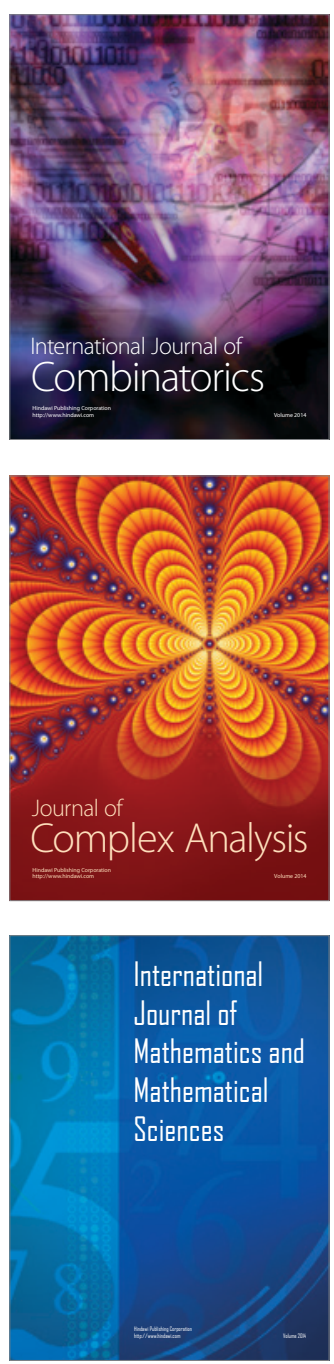
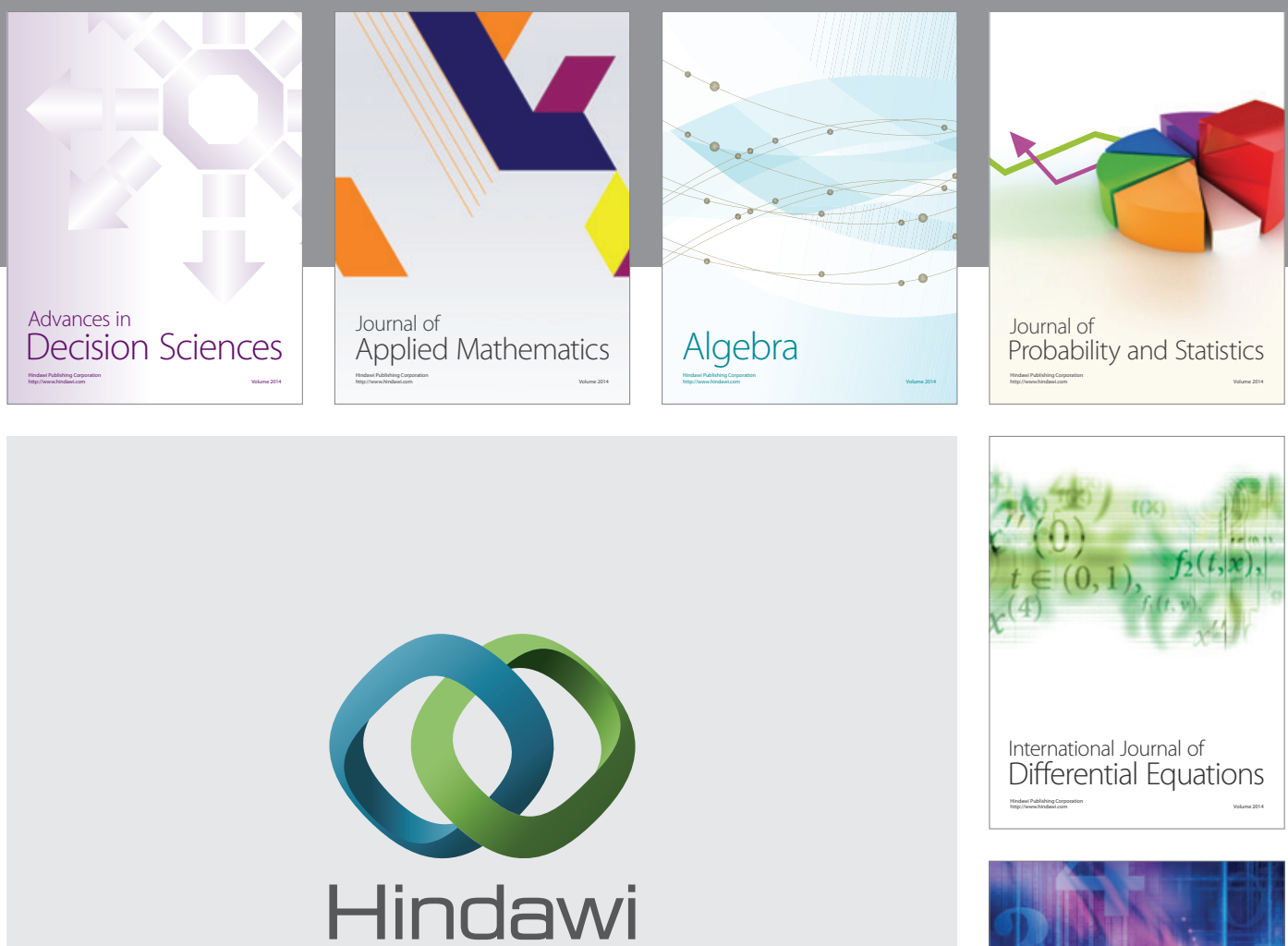

Submit your manuscripts at http://www.hindawi.com
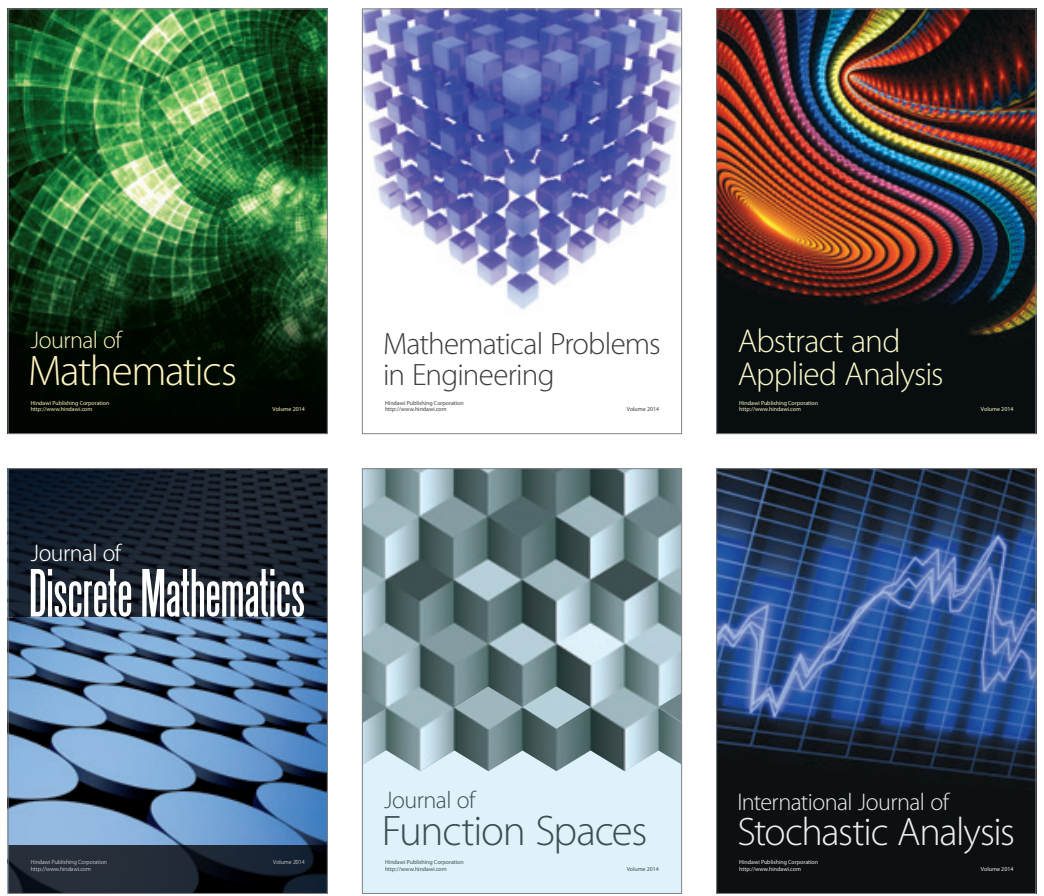

Journal of

Function Spaces

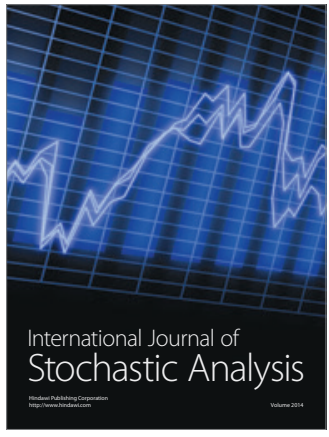

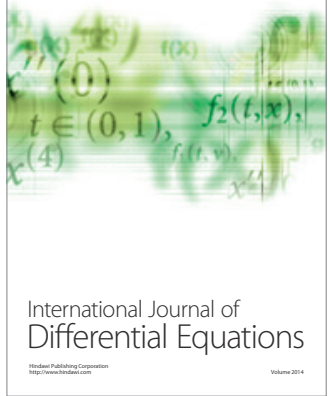
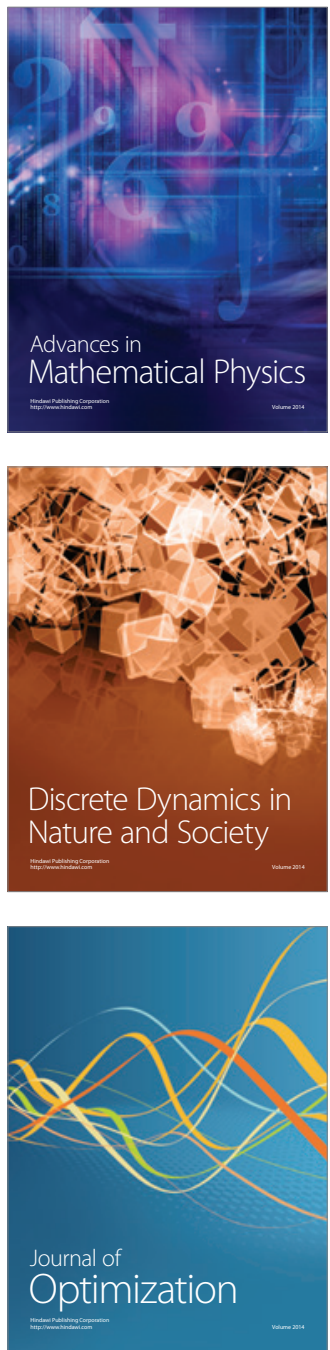